\title{
A Review of Electrospun Conductive Polyaniline Based Nanofiber Composites and Blends: Processing Features, Applications, and Future Directions
}

\author{
Saiful Izwan Abd Razak, ${ }^{1,2}$ Izzati Fatimah Wahab, ${ }^{2}$ Fatirah Fadil, ${ }^{3}$ \\ Farah Nuruljannah Dahli, ${ }^{4}$ Ahmad Zahran Md Khudzari, ${ }^{1,2}$ and Hassan Adeli ${ }^{5}$ \\ ${ }^{1}$ IJN-UTM Cardiovascular Engineering Centre, Institute of Human Centered Engineering, Universiti Teknologi Malaysia, \\ 81310 Skudai, Johor, Malaysia \\ ${ }^{2}$ Faculty of Bioscience and Medical Engineering, Universiti Teknologi Malaysia, 81310 Skudai, Johor, Malaysia \\ ${ }^{3}$ Department of Chemistry, Faculty of Science, Universiti Teknologi Malaysia, 81310 Skudai, Johor, Malaysia \\ ${ }^{4}$ Department of Bioprocess and Polymer Engineering, Faculty of Chemical and Energy Engineering, \\ Universiti Tun Hussein Onn Malaysia, 86400 Batu Pahat, Johor, Malaysia \\ ${ }^{5}$ Department of Chemical Engineering, University of Mazandaran, Babolsar, Iran \\ Correspondence should be addressed to Saiful Izwan Abd Razak; saifulizwan@utm.my
}

Received 18 October 2015; Revised 9 December 2015; Accepted 10 December 2015

Academic Editor: Giorgio Pia

Copyright (C) 2015 Saiful Izwan Abd Razak et al. This is an open access article distributed under the Creative Commons Attribution License, which permits unrestricted use, distribution, and reproduction in any medium, provided the original work is properly cited.

\begin{abstract}
Electrospun polymer nanofibers with high surface area to volume ratio and tunable characteristic are formed through the application of strong electrostatic field. Electrospinning has been identified as a straight forward and viable technique to produce nanofibers from polymer solution as their initial precursor. These nanofiber materials have attracted attention of researchers due to their enhanced and exceptional nanostructural characteristics. Electrospun polyaniline (PANI) based nanofiber is one of the important new materials for the rapidly growing technology development such as nanofiber based sensor devices, conductive tissue engineering scaffold materials, supercapacitors, and flexible solar cells applications. PANI however is relatively hard to process compared to that of other conventional polymers and plastics. The processing of PANI is daunting, mainly due to its rigid backbone which is related to its high level of conjugation. The challenges faced in the electrospinning processing of neat PANI have alternatively led to the development of the electrospun PANI based composites and blends. A review on the research activities of the electrospinning processing of the PANI based nanofibers, the potential prospect in various fields, and their future direction are presented.
\end{abstract}

\section{Introduction}

Nanostructures, structures of 100 nanometers (nm) or less in size, have been at the forefront of much modern engineering and science research due to their novel, significantly improved biological, chemical, and physical properties. There are mainly four types of nanostructures: zero- (0D) (e.g., nanoparticles), one- (1D) (e.g., nanowires, nanotubes, and nanofibers), two- (2D) (e.g., films and layers), and three- (3D) dimensional structures (e.g., polycrystals). Among them, one-dimensional (1D) nanostructures have been in the focus of quite extensive studies worldwide, because they represent the smallest dimensional structures with high aspect ratios and degrees of flexibility. Hence, these 1D nanostructures can be exploited as elements in nanodevices with various applications, such as supercapacitors, chemicals, and biosensors, in optoelectronics and tissue engineering [1-7].

Nanoapplication and nanotechnology development continues to stir the scientists' and engineers' interest in exploring the best combination of materials and $1 \mathrm{D}$ nanostructures. A few examples of such combinations are carbon nanotubes, inorganic semiconductor nanowire/nanobelts, 


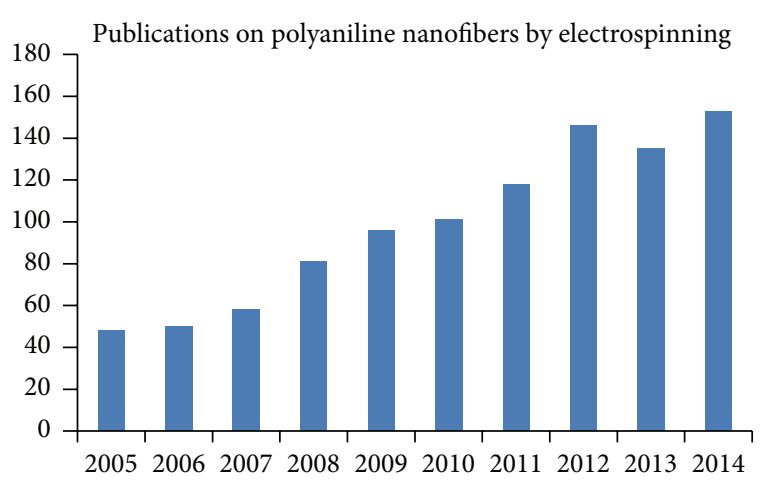

(a)

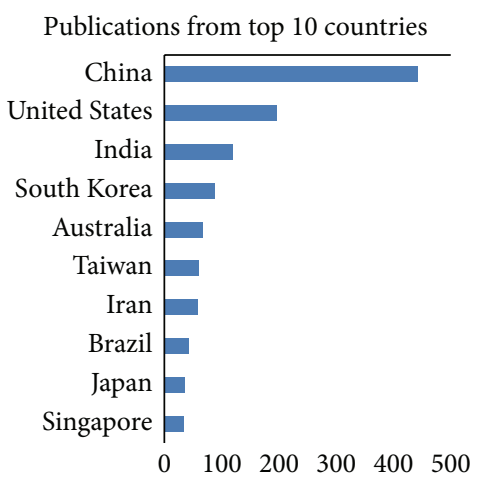

(b)

Figure 1: (a) Comparison of the annual number of scientific publications related to electrospinning polyaniline nanofibers. (b) Publication distribution around the world based on data analysis carried out using the Scopus search system with the term "polyaniline nanofibers" as of 30 March 2015.

metallic nanotubes/nanowires, and conjugated polymer nanofibers/nanotubes. The conjugated polymer 1D nanostructures possess certain highly attractive characteristics, such as an easily controllable bandgap, high mechanical flexibility, and greater biocompatibility than that of many inorganic materials. In addition, conjugated polymers are also called conducting polymers; in their neutral states their conductivity typically ranges from $10^{-10}$ to $10^{-5} \mathrm{Scm}^{-1}$. However, the conductivity can be enhanced into semiconductor or conductor states via chemical or electrochemical redox reactions. Among the various classes of conducting polymers, polyaniline (PANI) is one of the most widely investigated materials due to its easy synthesis, excellent optical and magnetic properties, and environmental stability. Furthermore, the electrical properties can be controlled by the oxidation and protonation state [8-10].

Up to now, a large number of approaches have already been demonstrated for preparing PANI 1D nanostructures, including chemical routes, such as hard physical templateguided synthesis and soft chemical template synthesis (interfacial polymerization, template-free method, dilute polymerization, and reverse emulsion polymerization), and a variety of lithography techniques [11]. However, the drawbacks of these techniques, such as required postsynthesis process, relatively poor control of the size and morphology uniformity, poorly oriented nanostructure arrays, and high costs, may limit their production on a large scale. Thus, physical approaches such as electrospinning are more advantageous compared to the above-mentioned techniques, especially for the mass production of continuous nanofibers.

\section{Electrospinning Theory and Principle}

Electrospinning is a fiber-spinning technology used to produce long, three-dimensional, ultrafine fibers [12]. According to Frenot and Chronakis, nanofibers produced by the electrospinning technique have diameters in the range of $100 \mathrm{~nm}$ to $1 \mu \mathrm{m}$ and lengths of up to kilometers [13]. Electrospun nanofibers present high attractiveness for diverse applications, such as in biomedical engineering and tissue regeneration [14], sensory and electronic devices [15, 16], smart textiles [17], and membrane filtration technology [18]. The viability of this technology is evidenced by the easiness of the spinning procedure and the simplicity in the custom setting of the electrospinning machine [19]. The most amazing characteristics of electrospun nanofibers include high surface area-to-volume ratio of well-interconnected nanofiber webs, flexibility in surface functionalities, tuneable surface properties, high permeability, and good mechanical performance [20].

The principle of the electrospinning technique was first described by Zeleny in his published work on the behavior of fluid droplets at the end of metal capillaries [12, 21]. Later on, in 1934, Formhals established the commercialized system of electrospinning for the fabrication of textile yarns in the U.S. Patent number 195704 [22]. Further development of the electrospinning from a polymer melt rather than a polymer solution using an air-blast to assist the fiber formation was patented by Norton in 1936 [23]. The conceptual principle of electrospinning is based on the employment of the external electric field to induce polymer fluid transformation by the elongation and whipping of the jet. In 1969, Taylor introduced the concept of Taylor cone, which is associated with the deformation of a liquid surface into a characteristic shape induced by electric fields. The theoretical analysis of the disintegration of liquid drops under electric fields, the calculation of the electric force acting on fine jets, of the critical voltage for inducing jet elongation, and of the voltage values at which Taylor cones are formed have been described in detail elsewhere [24]. Recently, the electrospinning process has gained huge popularity and the publication of works related to electrospinning increased over the years. A survey of the publications related to electrospun PANI nanofibers in the past 10 years is given in Figure 1(a), whereas the distribution of these published works among the 15 top countries is shown in Figure 1(b). These literature data were obtained based on the Scopus search system.

The data clearly demonstrate the progression of research works associated with PANI nanofibers obtained by means of electrospinning. The increase in the number of patents for diverse applications gives an insight into the recognition of 
the electrospinning process. A few companies, such as Espin Technologies, Nano Technics, and KATO Tech, are constantly benefited by the utmost features of nanofibers derived by electrospinning, while companies such as Donaldson Company and Freudenberg have already applied the electrospinning process in their air filtration products $[25,26]$.

In this report, a comprehensive review is made on the research and developments related to PANI electrospun nanofibers, including a discussion of their processing characteristics, physical and mechanical properties, and applications. Other basic issues regarding the processing limitations of the materials and research challenges are also considered in detail.

\section{Technical Aspects in Electrospinning}

From the technical point of view, the electrospinning equipment is an inexpensive, robust, and straight forward nanofiber production machine. The standard laboratory electrospinning unit generally consists of a metallic capillary needle, which is connected to a high voltage power supply and a grounded collector. The droplet of the spinning solution is discharged from the capillary needle in a continuous flow and the feed rate of the discharged droplet is adjusted using an infusion syringe pump. The electric field is applied at the tip of the capillary needle attached to the syringe, which is loaded with a spinning polymer solution, to induce an electric charge on the surface of the hanging droplet. The hanging droplet of the spinning solution, which is held by its surface tension, will then develop mutual charge repulsion due to excess pressure. The contraction of the surface charges to the counter electrode is caused by a force directly opposite to the surface tension of the liquid droplet [27].

The hemispherical droplet gets distorted into a conical shape, also called Taylor cone, under the simultaneous actions of the repulsive force. Additional increases in the electric field intensity will lead to reaching a critical value, whereby the repulsive electrostatic force overcomes the surface tension of the charged droplet to initiate the polymer jet formation. The initiation of the polymer jet then undergoes an instability and elongation process simultaneously with the evaporation of the solvent.

A grounded collector, which receives the deposition of the electrospun nanofibers from the charged jets, is placed at a certain distance from the metallic capillary needle [20]. A schematic diagram of the electrospinning system with the basic setup of a syringe pump attached to a disposable syringe with a metallic needle, a high voltage power supply unit, and a collector is Figure 2.

Multiple aspects of the electrospinning process have been demonstrated to affect the morphology of the nanofibers [27]. The major factors involved in the electrospinning process, including the polymer solutions, needles, and collectors, are discussed below [20].

3.1. Polymer Solutions. When dealing with the spinning solution of various polymers, the spinnability of the polymer solution becomes an utmost issue. The spinnability of a

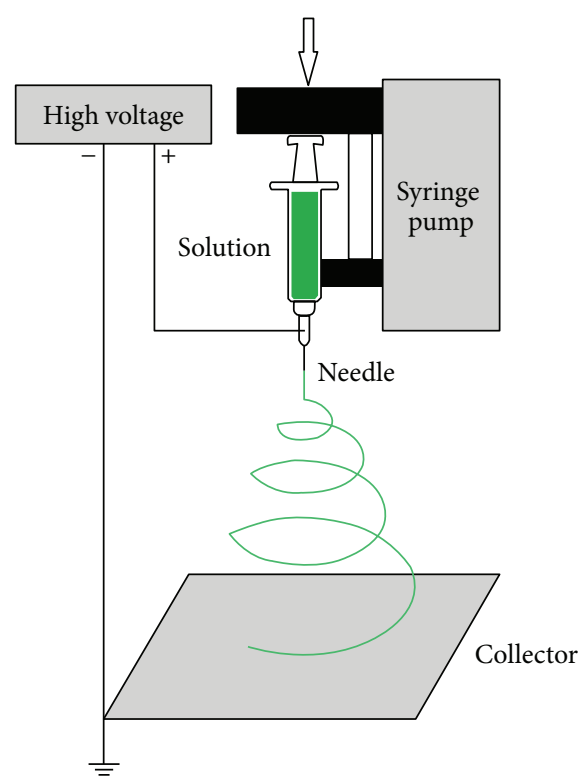

Figure 2: Schematic diagram of an electrospinning setup.

solution depends on several aspects during the electrospinning process [28]. These parameters can be classified into major and minor influences. Major parameters comprise the viscosity, elasticity, and surface tension of the solution, the hydrostatic pressure of the solution in the capillary needle, the electric potential, and the distance from the capillary tip to the collector, while ambient influences, such as solution temperature, humidity, and air velocity, can be classified as minor parameters [29].

The difference in the viscosities and surface tension of the spinning solution will directly influence the morphology of the nanofibers formed either as an ultrathin or as beaded fibrous structure. A spinning solution with viscosity values of 1-20 poises and surface tension values of 35-55 dynes $/ \mathrm{cm}^{2}$ is regarded as a suitable solution for inducing fiber formation. Meanwhile, at viscosities of above 20 poises, the electrospinning is hindered because of the flow instability caused by the high cohesiveness of the solution. As the viscosity of the solution is increased, the beads become larger, the average distance between beads is longer, and enlarged fiber diameters are observed. The formation of beaded fibers occurs because the fluid jet breaks up to droplets when the viscosity of the spinning solution is below 1 poise [30].

The need for a volatile solvent as carrier of a particular polymer in solution spinning is one of the fundamental criteria in electrospinning. Solvent selection is of paramount importance in determining the critical minimum solution concentration to allow the transition from electrospraying to electrospinning, thereby significantly affecting solution spinnability and the morphology of the electrospun nanofibers [31]. Bahrami and Gholipour Kanani studied the effects of using solvents of different polarity, such as glacial acetic acid, dimethyl formamide, glacial formic acid, and acetone, on the morphology of polymer nanofibers. It was reported that the use of glacial acetic acid as solvent resulted 

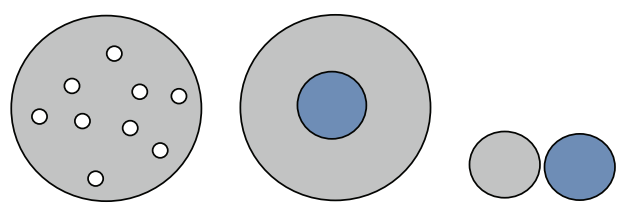

$\square$ Polymer A

(a)

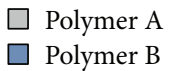

(b) $\square$ Polymer A

(c)
FIGURE 3: Schematic cross-sectional view of different structures of composite nanofibers: (a) randomly blended; (b) core-shell; (c) mingled structure generated from various capillary needle nozzles.

in the formation of fibers with nonuniform diameter distribution compared to other solvents. This was due to the high dielectric constant value of acetic acid [32]. Generally, the net volume charge density is proportional to the conductivity of the solution. As the net charge density increases, the beads become smaller and change more into a spindle-like shape, while the fibers become thinner. The neutralization of the charge carried by the polymer jet favors the formation of beads, as the tension in the fiber depends on the net charge repulsion and the interaction of the net charge with the electric field [30]. One of the effective approaches to adjust the solution conductivity is by introducing a conductive nanoparticle filler or soluble salt into the solution. The incorporation of salt increased the stretching of the polymer jet and reduced the incident of bead formation by distributing the charges consistently to control the fiber uniformity [33].

3.2. Needles. Typical electrospun nanofibers represent solid fibers, with a smooth surface and circular cross section. However, electrospun nanofibers can also be designed and fabricated in hybrid form of either basically random blending with nanoparticles or ordered structures, such as core-sheath and mingled nanofibers, which consist of two distinctive polymers. Various designs of electrospun nanofibers were successfully created by the use of different needles (coelectrospinning). These needles allow the formation of different fiber morphologies that could be exploited for specific uses [34]. Cross-sectional views of electrospun nanofibers in the forms of randomly blended structure, core-shell structure, and mingled structure are illustrated in Figure 3.

For the randomly blended nanofibers, a standard needle with only one capillary opening is used. However, core-shell and mingle structured nanofibers need to be spun using a special needle, respectively, as shown in Figure 4. For the core-sheath needle, two capillary openings are combined: the outer capillary opening is for the sheath and the inner capillary opening is for the core, while for mingled structured nanofiber formation, the use of a coaxial needle with double or multiple capillary openings in a parallel axis is needed.

Nonetheless, the same difficulties in optimizing the solution properties are still present and affecting the multineedle design of the electrospinning system. However, since the throughput is not a primary concern in this case, interesting nanofiber structures can be obtained if the nonuniform deposition of the fiber can be taken under control [35]. Bicomponent mingled structured fibers have been obtained by merging side-by-side two spinnerets fed by separate reservoirs [36, 37]. In spite of the fact that the electrospinning of polymer blends can be achieved by spinning a mixture of polymers or polymer solutions through a single needle, the electrospinning setup can be designed as having two separate reservoirs to avoid some limitations related to the physics of polymeric blends. Indeed, with a single spinneret, a homogenous, thermodynamically stable mix of polymers is required and, in the case of solution electrospinning, the interactions between the polymer and the solvent of the opposing pair become crucial. With two separate spinnerets, the polymer solutions will come in contact only at the end of the spinning head, allowing for the formation of blend fibers that are otherwise difficult to produce [35].

3.3. Collectors. The collector used during the electrospinning process would also have an influence on the morphology of the electrospun nanofibers. At first, the randomly oriented nanofibers were obtained by collecting the fibers from a stationary collector covered with aluminium foil [38]. An attempt to obtain the deposited nanofibers in aligned orientation has been made as the electrospinning jet was shown to be sensitive to small differences in the electric field profile [39]. A conductive collecting substrate with textured surface, such as wire mesh or grid, has been used to form patterned/textured nanofibers due to its electric field profile. The results show that, in a typical square grid substrate, the nanofibers are preferentially deposited along the wire. As the wires forming the grid are narrow, nanofibers tend to align along the length of the wire. Despite the preferential deposition of fibers on the wire, the presence of numerous neighbouring wires often diverts the electrospinning jet to the adjacent wire. This results in a higher density of the nanofibers that are aligned on the wire, compared to the randomly oriented, scattered nanofibers at the gaps between the wires. Where the distance between the wires gets larger, the difference in the density of the nanofibers on the wires as well as the space between the wires also increases. There are differences in fiber diameter as well whereby the nanofibers deposited on the wire are larger than the nanofibers deposited within the space between the wires. This has been attributed to a greater stretching of the nanofibers when they span across the space [40]. Other forms of patterned substrates have also been used to collect the deposited nanofibers. Grids made out of parallel wires close to one another have been shown to form dense, aligned nanofibers between the wires, while a substrate with arrayed pins gives rise to a membrane made out of radiating nanofibers between the pins [41]. More grid variations, such as having a pin in the center of a round space, form a pattern where fibers radiate from the central pin to the edges.

Furthermore, aligned nanofibers have also been successfully collected by using a rotating disk collector instead of the stationary collector. The adjustment of the take-up velocity of the rotating disk collector proved that the fiber alignment was a direct function of the velocity of the rotating disk. Adding to this, as the take-up velocity increased from $630 \mathrm{~m} / \mathrm{min}$ 


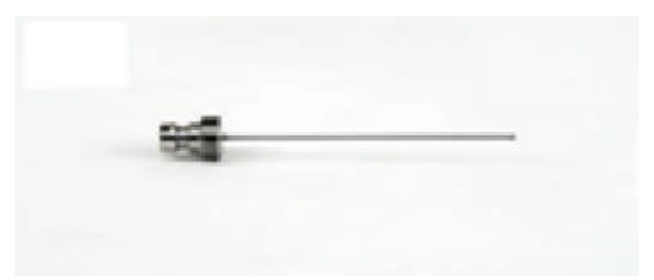

(a)

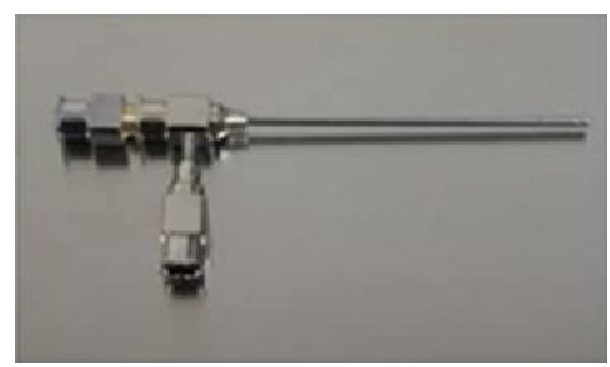

(c)

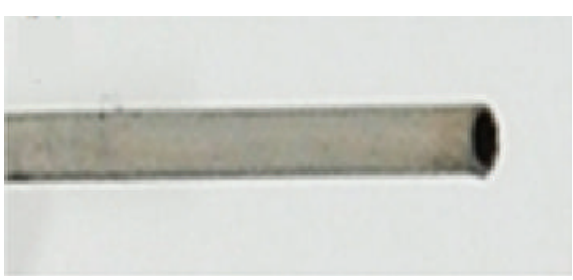

(b)

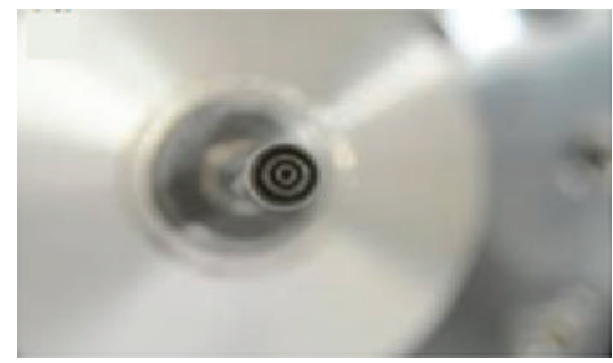

(d)

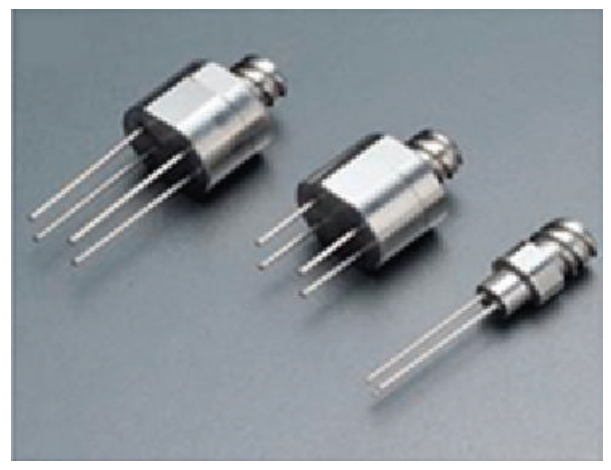

(e)

FIGURE 4: Various needles used in electrospinning: (a) standard needle; (b) inlet of standard needle; (c) core-sheath needle; (d) inlet of coresheath needle; (e) coaxial needle with double or multiple capillary openings.

to $1890 \mathrm{~m} / \mathrm{min}$, the average diameter of aligned nanofibers decreased from 700 to $350 \mathrm{~nm}$ [42].

3.4. Configurations. There have been established several types of arrangement of the electrospinning setup, such as horizontal, vertical upward, and vertical downward. For each type of arrangement, the positioning of the components is different. Figure 5 illustrates the arrangement of the electrospinning in detail. For a small laboratory scale use, the vertical downward setup of the electrospinning is the most favorable due to the simple optimization and operational monitoring.

Most of the research works on the electrospinning nanofibers completed in the universities are based on the conventional single needle. One of the main issues to overcome in this case is the limited throughput obtainable by the use of such a system. For industrial applications, despite the commonly high added value of the products, the low production rate (in the order of tenths of grams per hour) of the basic electrospinning apparatus represents a severe restraint. Therefore, the mass production of the nanofibers by using the conventional single needle is tough and challenging. For this reason, the vertical upward setup has been selected for the industrial production scale. This employs a new design for supplying the solution to a metal roller spinneret. The advantage of this setup is its ease of scaling-up for increased output. By using the needleless electrospinning system, the possibilities to increase the production rate of nanofibers are higher due to the use of multiple jets. The productivity rate has been significantly enhanced within 30 times higher than that of the single needle electrospinning [43].

The setup of the needleless electrospinning system was firstly introduced in 2010 by Tang et al., whereby the nanofibers could be produced in abundance by applying the method of splashing the polymer solution onto the surface of a metal roller spinneret [44]. In this method, the setup consisted of a metal roller spinneret as the positive electrode connected to a high voltage power supply. The polymer solution droplets were splashed onto the surface of the metal roller spinneret through the holes of the solution distributor, which was located above the spinneret. Niu et al. [45] improvised the system by inventing a spiral coil setup and proved that this method had a higher fiber production rate and allowed better control of fiber morphology. Considering the above information, one can conclude that various designs and 


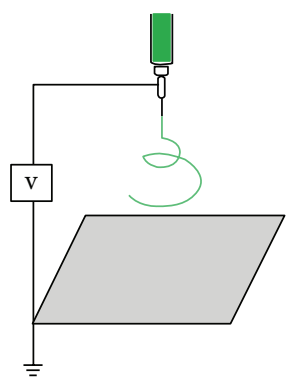

(a)

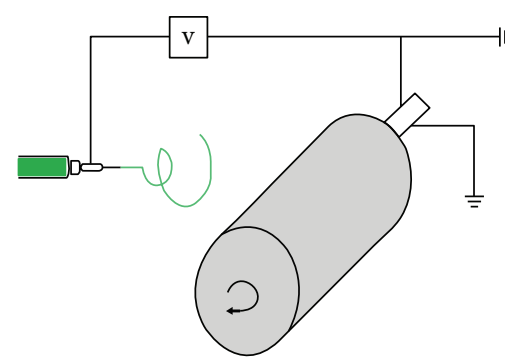

(b)

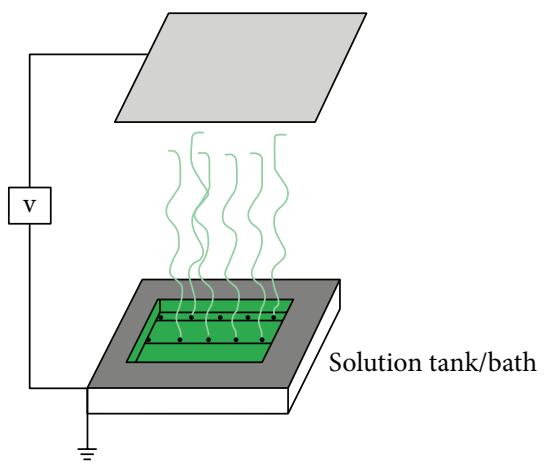

(c)

FIGURE 5: Common configuration of electrospinning: (a) vertical; (b) horizontal; (c) needleless.

dimensions of nanofibers can be tailored by using different kinds of electrospinning setup.

\section{Basics of PANI}

Conducting polymers are the fourth generation of polymeric materials. This type of nanofibers has become the source for the development of films, membranes, and nanoelectrodes for sensor applications. Their electrical conductivities can be increased by many orders of magnitude from $10^{-10}-10^{-5}$ to $10^{2}-10^{5} \mathrm{Scm}^{-1}$ upon doping $[8,46]$, which covers the whole insulator-semiconductor-metal range. A variety of conducting polymers, such as polyaniline (PANI), polypyrrole (PPY), poly(p-phenylene-vinylene) (PPV), poly(3,4-ethylene dioxythiophene) (PEDOT), and other polythiophene derivatives, which can be synthesized into 1D nanostructures, such as nanotubes and nanowires, have recently attracted attention in the areas of nanoscience and nanotechnology due to their special conduction mechanism, unique electrical properties, reversible doping/dedoping process, controllable chemical and electrochemical properties, and processability [46-48].

PANI has the longest history among the intrinsically conducting polymers. It is one of the oldest artificial conducting polymers and its high electrical conductivity among organic compounds has attracted continuing attention. PANI has become one of the most attractive conducting polymers due to its high stability, ease of synthesis, feasibility of electrical conductivity control by changing either the protonation state or the oxidation state, and the low cost of the aniline monomer [49-51]. PANI is either totally insulating or electrically conductive, depending on the oxidation state and protonation level. Only in the intermediate oxidation state, the protonated emeraldine form is conductive. The fully reduced leucoemeraldine and fully oxidized pernigraniline are insulating materials [52]. PANI nanofibers have received much attention due to their superior properties compared to the conventional bulk PANI or PANI film [53-55]. PANI nanofibers show enhanced water processability [56] and improved acid-base sensitivity and response time when they are exposed to chemical vapor, due to their large surface area. On the other hand, PANI nanofibers have numerous potential applications, including electric devices, flash welding [55], sensors and actuators [57, 58], rechargeable batteries [59], electromagnetic shielding devices, and anticorrosion coating [60].

The neutral intrinsic redox states of PANI can vary from that of the fully reduced leucoemeraldine to that of the fully oxidized pernigraniline. The $50 \%$ intrinsically oxidized polymer has been termed emeraldine and the $75 \%$ intrinsically oxidized polymer is called nigraniline [61]. Unlike most other polyaromatics, the fully oxidized state of PANI is not conducting. PANI becomes conducting when the emeraldine base is protonated and charge carriers are generated. The process is generally termed protonic acid doping. The green protonated emeraldine salt has conductivity by many orders of magnitude higher than that of common polymers, but lower than that of typical metals.

PANI is composed of the repeating units of the aniline monomer connected to form a backbone. The existence of a nitrogen atom lying between the phenyl rings allows the formation of different oxidation states, which can affect its physical properties. PANI has an inherently unstable backbone, resulting from the formation of alternate single and double bonds along the monomer units during polymerization. The delocalized $\pi$ bonding electrons, produced across the conjugated backbone, provide an electrical pathway for mobile charge carriers, which are introduced through doping. Consequently, the electronic properties of PANI, as well as many other physicochemical properties, are determined by the structure of the polymer backbone and the nature and concentration of the dopant ions [62-64].

4.1. PANI Nanostructures. Much effort has been put in synthesizing nanostructured PANI, owing to its novel physical properties and potential applications. Generally, PANI nanostructures can be synthesized using hard- and softtemplate methods. In the hard-template method, which typically employs an aluminium oxide or track-etched membrane as a template for synthesizing the desired material, polymerization occurs within the pores of the membrane [65]. However, a rather tedious postsynthesis process is often required to remove the template used [66]. Additionally, 
preformed PANI nanostructures may be destroyed or form undesirable aggregates when released from the template. The soft-template method uses micelles, emulsions, liquid crystals, and surfactant gels to synthesize PANI nanostructures [67].

The soft-template method $[68,69]$ is a simple self-assembly method. By controlling the synthesis conditions, including the temperature and molar ratio of monomer to dopant, PANI nanostructures can be prepared by in situ doping polymerization in the presence of protonic acids as dopants. In the self-assembled formation mechanism using this approach, the micelles formed by dopant and/or monomer dopant act as soft templates in the process of forming tubes/wires [68]. Up to now, a variety of PANI micro/nanostructures, such as micro/nanotubes [70, 71], nanowires/fibers [72-74], hollow microspheres [75, 76], nanotube junctions, and dendrites [77], have been prepared by the soft-template method. However, this method allows for a limited range of chemicals to be used. Moreover, to date, some novel template-less methods have also been developed to shape PANI into nanofibers, such as electrospinning [78]. The nanofibers can be synthesized by deposition directly onto aluminium foil, wire mesh, cotton, silk, wool, and Si wafer.

Many other methods have also been recognized for the synthesis of PANI nanostructures. PANI films with a thickness of 20 to $100 \mathrm{~nm}$ have been prepared using vaporphase polymerization [79]. Vapor-phase polymerization is one of the self-assembling techniques that do not require a host polymer. It utilizes the organic arrangement of macro molecules to fabricate nanolayered aggregates and thin films $[80,81]$. There are also reports on the preparation of nanostructured PANI through polymerization in the presence of functionalized protonic acid, which acts as both surfactant (and emulsifier) and protonating agent. This process is called emulsion polymerization and it combines aniline, protonic acid, and oxidant with a mixture of water and a nonpolar or weakly polar liquid [82-84]. In order to avoid agglomeration of thick fibers into irregularly shaped particles, Jing and coworkers have employed ultrasonic radiation to assist the conventional dropwise addition of the oxidant solution to form PANI nanofibers [85]. Ultrasound assistance through the sonochemical method is an important approach, allowing researchers to obtain uniform dispersion of inorganic nanoparticles in the polymer matrix [86, 87]. Another popular method to obtain PANI nanofibers is through interfacial polymerization, which involves step polymerization of two reactive agents or monomers that dissolve in two immiscible phases. This technique allows the synthesis of high crystallinity PANI nanofibers without depending on specific templates and solvents [60,88-90].

\section{PANI Nanofibers via Electrospinning}

5.1. Neat PANI Nanofibers. Producing neat PANI nanofiber by electrospinning has been a great challenge for researchers. The poor solubility of PANI in common solvents interferes with electrospinning into uniform fibers, requiring it to be doped by organic acids in order to increase its solubility in organic solvents [91]. Various methods have been attempted to test suitability of electrospinning PANI solutions without blending with other polymers. Being an intrinsically conducting polymer, PANI is available only in the form of low molecular weight, which thus makes the elasticity of its solution insufficient for being electrospun directly into a fiber form [79]. Previous studies reported the formation of structures like beads and droplets in electrospun fibers with low elasticity [92]. The use of an insulating copolymer with high molecular weight is expected to act as a remedy, imparting greater elasticity. However, the presence of a nonconducting polymer strongly modifies the physical and chemical properties of PANI and eventually limits its applications [93]. The fabrication of one-dimensional polymer field effect transistor using PANI nanofibers blended with polyethylene oxide $(\mathrm{PEO})$ in a 1:1 ratio has been reported. The addition of $\mathrm{PEO}$ resulted in a drastic decrement of conductivity. The results of the study allow the conclusion that reducing the PEO content will enhance the conductivity of the electrospun PANI by one or several orders of magnitude [94].

In order to improve the electrospinning processing of PANI, a few routes have been developed to find the optimum conditions to produce pure PANI nanofibers with smooth fiber surface and to minimize the limitations caused by nonconducting copolymers. The objective was not only to produce neat PANI, but also to obtain fibers that would raise no concern about side reactions with the doping agents when exposed to external agents, such as radiation or gases [93]. In their study, Cárdenas and coworkers proposed using a bath collector in the fabrication of PANI nanofibers by the electrospinning procedure. In this research work, the fibers were not deposited on the grounded collector, but instead they were collected in an acetone bath placed on an electrode [95]. Although there are a number of literature sources [9698] describing the preparation of PANI based nanofibers by electrospinning, the reports on the fabrication of neat PANI nanofibers by electrospinning are quite rare. In an earlier study, MacDiarmid et al. [99] produced nanofibers of PANI by a method similar to the one used by Cárdenas et al. and achieved fibers with an average diameter of $139 \mathrm{~nm}$ by placing a $20 \mathrm{wt} \%$ solution of PANI in $98 \%$ sulfuric acid in a glass pipette fixed above a copper cathode immersed in pure water. However, in contrast to this work, Cárdenas and coworkers used a solution with a considerably lower concentration of PANI (1 wt\%) in acetone [95].

By using the acetone bath as collector, the excess solvent was diffused into acetone and allowed fiber formation. Thus, the researchers have successfully fabricated neat submicron PANI using the electrospinning technique without incorporation of high molecular weight polymer blending for gaining jet stability to form fibers. The fibers produced by this technique were observed and characterized under scanning electron microscope (SEM). These fibers appeared as isolated on the substrates, which is favorable for electrical characterization. The conductivity of these fibers was measured to be in the order of $10^{-3}$ to $10^{2} \mathrm{Scm}^{-1}$, with a significant increasing trend in the conductivity values. Nanofibers with larger diameters have higher values of conductivity due to their increased volume to surface ratio. A higher volume 
to surface ratio causes a relatively slower loss of solvent by evaporation and consequently the fibers will be more partially doped and conductive.

The method of utilizing the copper cathode immersed in pure water was done by placing $20 \mathrm{wt} \%$ of PANI solution with $98 \%$ sulfuric acid inside the glass pipette. The fibers were collected either in or at the surface of water. In this study, the authors reported having successfully produced fibers with average diameters of $139 \mathrm{~nm}$, the conductivity of single fibers being of about $\sim 0.1 \mathrm{Scm}^{-1}$. It is noticeable that the morphology of the nanofibers deposited in the solution bath was less smooth as compared to that of the nanofibers collected from the conductive surface [99].

Another study by Shie et al. [100] reported the use of the emeraldine base of poly(o-methoxyaniline) powder dissolved in a mixture of tetrahydrofuran/dimethylformamide (THF/DMF) for the preparation of the electrospinning solution. A $5 \mathrm{wt} \%$ solution of PANI was loaded into a plastic syringe connected with a metallic needle, with a rate of 0.02 to $0.04 \mathrm{~mL} / \mathrm{min}$. PANI nanofibers were collected on an aluminum plate placed 8 to $14 \mathrm{~cm}$ below the tip of the needle. The samples were then heated at $100^{\circ} \mathrm{C}$ for an hour. The biocompatibility of these fibers was examined through various analyses: agar diffusion test, nitric oxide assay, and cell and protein adsorption. The study found that the PANI nanofibers fabricated by electrospinning possessed negligible cytotoxicity, low serum protein absorption, and low myoblast cell attachment. The percentage of myoblast cell attachment to PANI was measured to be $40.7 \% \pm 6.1 \%$, while cell adhesion to electrically stimulated PANI was $53.6 \% \pm 9.9 \%$, which was slightly higher. The results indicated that the cells on the PANI fibers exhibited a significantly extended lag phase of growth, while after electrical stimulation, the cells demonstrated higher proliferation on PANI fibers. The decreased cell growth on PANI fibers was probably caused by the hydrophobic surface of the fibers. A significant increase of nitric oxide secretion by PANI nanofibers shows that these fibers may elicit a slight inflammatory response after implantation in the human body. The response index of the toxicity assay of PANI to myoblast cells was nearly zero after electrical stimulation. Thus, it is suggested that PANI nanofibers can be considered to be very suitable for biosensor applications.

A study of electrospun PANI doped with 2-acrylomido2-ethyl-1-propanesulfonic acid (AMPSA) indicated a conductivity of $0.76 \mathrm{Scm}^{-1}$, suggesting that the fibers were in the metallic regime of charge transport since there was no apparent field effect [101]. Pure PANI fibers were prepared by dissolving $900 \mathrm{mg}$ of PANI-AMPSA into $3 \mathrm{~mL}$ concentrated sulfuric acid and manually stirring the solution with a glass rod for $30 \mathrm{~min}$. The solution was then held in a glass pipette placed vertically $3 \mathrm{~cm}$ over a beaker of water acting as cathode. A value of $15 \mathrm{kV}$ of spinning voltage was successfully applied, leading to the deposition of fine fibers in an erratic manner due to the spark formation at the end of the pipette. Fibers deposited on the surface of water and were left in the water for $12 \mathrm{~h}$ to allow the sulfuric acid to dissolve in the water. The fibers were then collected on a degenerately doped $\mathrm{Si} / \mathrm{SiO}_{2}$ wafer. The average diameter of these fibers was measured to be approximately $10 \mu \mathrm{m}$.

5.2. Blend PANI Nanofibers. Since PANI is difficult to be electrospun, most researchers tend to incorporate it with other polymers to make it electrospinnable. Chen and coworkers studied the incorporation of PANI into poly( $\varepsilon$-caprolactone) and gelatin for orthotopic photothermal treatment of tumors in vivo [102]. Apart from having the right polymer candidates to be blended with, the solvent used during the electrospinning solution preparation is also an important parameter to consider. Fryczkowski et al. investigated the effect of two different solvents in preparing electrospinning solution of poly(3-hydroxybutyrate) (PHB) with PANI [103]. Their study showed potential biodegradability and interesting electrical properties for PHB-PANI. THF gave better nanofiber structure and properties than chloroform. PHBPANI in THF solvent produces smooth, uniform, and fine nanofibers with enhanced hydrophilicity and low electrical resistance.

Karim utilized dual approach to incorporate PANI aligned nanofibers [104]. The first step was to synthesize PANI copolymer with o-aminobenzenesulfonic acid (PANI-coPABSA) by in-situ polymerization. Then, polyvinyl alcohol (PVA) and chitosan oligosaccharide (COS) were blended into the PANI copolymer to make the electrospinning solution. XRD and FTIR data from this study displayed the existence of hydrogen bonds between PANI-co-PABSA, COS, and PVA molecules that may possibly cause weak interaction in COS.

Sharma et al. made use of electrospun fibers to fabricate scaffolds for three-dimensional cell culture [105]. They reported fabrication of $\mathrm{PANI} /$ poly $(\mathrm{N}$-isopropyl acrylamideco-methacrylic acid) (PANI-CNT/PNIPAm-co-MAA) composite by $1: 1$ ratio of polymers. This study suggests possible use of conducting nanofibers as scaffolds after obtaining better cell survival than the control and PNIPA-co-MAA without PANI. An effort to replace high cost platinum for oxygen reduction reaction catalyst in polymer electrolyte fuel cells with cheaper material has been done by Zamani et al. by having metal-polymer blend [106]. $10 \mathrm{wt} \%$ PANI addition into polyacrylonitrile (PAN) was firstly used as nanofiber catalysts and it provides improvements to half-wave potential and ORR onset potential by 70 and $100 \mathrm{mV}$.

PANI nanofibers with the least amount of PEO, within the range of 1 to $0 \% \mathrm{w} / \mathrm{w}$ of $\mathrm{PEO}$ content, with respect to the amount of PANI, revealed some defects such as bead formation [94]. Such drawbacks could be explained by the capillary instability of the spinning jet by surface tension as PEO concentration was decreased [107]. Compared to PEO, polystyrene (PS) was found to be more suitable for blending with PANI, as it led to the formation of thinner PANI nanofibers, with a diameter below $100 \mathrm{~nm}$. Thus, electrospinning the blended polymer solution produced nanofibers with fewer defects, as compared to PEO, and presented better homogeneity of the blend in chloroform. Since the conductivity of PANI is very sensitive to the amount of defects, it is reasonable to suggest that the overall conductivity of the PANI/PS blends will be higher than that of the PANI/PEO blends [91]. 
5.3. Core/Shell PANI Nanofibers. As mentioned earlier, coaxial spinning can also be used in producing PANI nanofibers. This kind of electrospinning uses two spinnerets, which allows the low elastic fluid to elongate along with the electrospinnable fluid [108]. This process will result in nanofiber with continuous core-sheath morphology. Zhang and Rutledge produced fibers of PANI with a dopant of camphor-10-sulfonic acid (HCSA) blended with poly(methyl methacrylate) (PMMA) in core-sheath form through coaxial electrospinning. 100\% doped-PANI fibers were then obtained by immersing the fiber blend into isopropyl alcohol. This was done to remove the PMMA shells and thereby release the doped PANI cores. Due to the removal, the diameters of the fibers decreased from $1440 \pm 200$ to $620 \pm 160 \mathrm{~nm}$ [109].

Coaxial electrospinning is also useful in incorporating conducting material onto nonconducting fibers (or vice versa) with distinct layers. Sarvi et al. applied this approach to develop nanofibers with superior piezoelectricity [110]. The nanofibers consist of poly(vinylidene fluoride) (PVDF) as the core and blends of PVA, PANI, and multiwalled carbon nanotube (MWCNT) as the shell. Low concentration of PVDF was added to PANI solution in order to increase the viscosity of relatively dilute PANI solution so that it is electrospinnable. MWCNT was reported to significantly reduce the electrical percolation concentration in the polymer matrix. It was shown that the presence of PANI component increased the PVA nanofibers mat formation by bridging the nanofibers and eliminating air resistance.

An attempt to develop a material extraction for in vivo, semisolid tissue extraction has been done by preparing polystyrene (PS)/PANI core-sheath electrospun nanofibers [111]. Wu et al. have previously worked on PS/crosslinked collagen core-sheath for same purpose but the PS core showed poor extraction ability to very polar and ionic compounds [112]. The collagen sheath failed to extract and therefore would prevent the permeation of tissue matrix into PS core. Later, they successfully modified the fabrication of core-sheath by having PANI sheath. Neat PANI sheath was obtained after selectively removed collagen content in as-spun collagen/PANI sheath. This approach gave better efficiency for acidic phytohormones and high extraction capacity.

5.4. Modified Core/Shell PANI Nanofibers. Various modifications can also be done to produce variations of core/shell structure. Spinneret of coaxial electrospinning can be designed to produce novel ribbon-shaped flexible nanocables [113]. The modified spinneret allows the core for the nanoribbon to be adjusted to achieve preferred diameters by changing the inner needle. This novel technique could fabricate tunable core composed of PANI, magnetic $\mathrm{Fe}_{3} \mathrm{O}_{4}$ nanoparticles, and PMMA template with photoluminescent $\mathrm{Tb}(\mathrm{BA})_{3}$ phen (BA = benzoic acid, phen =1,10-phenanthroline). The nanocable array produced electrically conductive-magnetic bifunctional core with insulating photoluminescent sheath. The conductive cores possessed electrical conductivity at the order of $10^{-2} \mathrm{Scm}^{-1}$ and may give response in magnetic field. These conductive nanocables were insulated with each other in the array form and able to emit green light under ultraviolet light due to the sheath properties. The ability to tune the emission colour of their belt-shaped coaxial microcables by changing the europium/terbium $\left(\mathrm{Eu}^{+} / \mathrm{Tb}^{+}\right)$molar ratio in the sheath was shown by Shao et al. [114]. They were able to tune the emission in wide range of red-yellow-green colour by adjusting the mass ratio of $\mathrm{Eu}^{+} / \mathrm{Tb}^{+}$complexes, PANI content, or $\mathrm{Fe}_{3} \mathrm{O}_{4}$ nanoparticles content.

An attempt by Liu et al. has led to an interesting way to fabricate PANI nanofiber with hollow structure through electrospinning [115]. Instead of using the conventional coaxial electrospinning, the technique did not require the preparation of two different electrospun solutions. The onepot electrospinning requires only one mixture of solution favored for the shell and eventually directly produces hollow electrospun nanofibers. Liu et al. fabricated trifunctional (photoluminescent-electrical-magnetic) flexible $\mathrm{Eu}(\mathrm{BA})_{3}$ phen/PANI/ $\mathrm{Fe}_{3} \mathrm{O}_{4} / \mathrm{PVP}$ hollow nanofibers. The hollow nanofibers had outer diameters of $305 \mathrm{~nm}$ and inner diameters of about $140 \mathrm{~nm}$. The team also used the same procedure to produce $\mathrm{Tb}(\mathrm{BA})_{3}$ phen/PANI/ $\mathrm{Fe}_{3} \mathrm{O}_{4} / \mathrm{PVP}$ hollow nanofibers with $238 \mathrm{~nm}$ outer diameters and $80 \mathrm{~nm}$ inner diameters [116]. There are also few studies that reported hollow structured PANI nanofibers. However, those nanofibers were obtained by the in situ polymerization of aniline on the surface of as-prepared electrospun nanofibers and followed by removal of the electrospun template [117].

$\mathrm{Lv}$ and coworkers applied electrospinning using specially designed parallel dual spinnerets to prepare bistrandaligned nanobundles [118]. These nanobundles consisted of PANI/PVP as one strand and $\mathrm{Eu}(\mathrm{BA})_{3}$ phen/PVP as another strand, where the PVP acted as template. They are the first to record this procedure of obtaining bistrandaligned nanostructures. They suggested better connectivity of PANI in the nanofibers and effective isolation of rare earth complex.

The mechanical properties of electrospun nanofibers are generally considered important for many applications. In spite of this, nanofibers are often found to be mechanically poorer than their corresponding textile fibers made from the same polymers. Their tensile strength and Young's modulus were reported below $300 \mathrm{MPa}$ and $3 \mathrm{GPa}$, respectively [119121]. To produce high-performance electrospun nanofibers, the molecular structures of the nanofibers should be oriented with chain extension along the fiber axis [122]. PANI on the other hand usually acts as conducting particulate filler in a suitable matrix, which provides the required mechanical properties. Valentová and Stejskal [123] characterized the mechanical properties of PANI on compressed circular pellets instead of conventional rectangular or dumbbell shaped samples. It was found out that the dynamic mechanical properties of the compressed pellets depended on the compression pressure. The samples became brittle at a compression pressure above $800 \mathrm{MPa}$. PANI fibers were wet spun from a solution of PANI containing solvent. It was found that the coagulant temperature had significant effects on the strength and modulus of the spun fibers [124]. 


\section{Applications of Electrospun PANI Nanofibers}

Researchers have been investigating the potential applications of electrospun PANI nanofibers in various fields. This section highlights opportunities of using electrospun PANI nanofibers for emerging technology development, such as in the conductive scaffolding materials, controlled drug delivery systems, nanofiber based sensor devices, supercapacitors, and flexible solar cells.

6.1. Tissue Engineering and Drug Delivery. Tissue engineering is a method to fabricate new tissue from cultured cells. Biodegradable scaffolds can act as transplant vehicles for cultured cells and templates to guide tissue regeneration [125, 126]. The scaffolds should be biocompatible, biodegradable, and highly porous; they should have large surface area and highly interconnected pore structure with suitable pore size $[127,128]$. PANI is one of the conducting polymers that have the potential to be applied as conductive substrates for tissue engineering [129-131]. It is only quite recently that the tunable electroactivity of PANI has been explored in the area of diverse biological applications, such as for scaffolds in the tissue engineering field. Still, the investigation of PANI for such applications has already brought evidence of the ability of PANI and blended PANI to support cell growth. The ability of varying the oxidative state of PANI has increased the research development in biomedical research fields [132].

Although, like many conductive polymers, PANI is not inherently biodegradable [133], it can be made so by (1) blending it with a biodegradable polymer to form a composite [134]; (2) modifying the backbone to render degradability [135]; and (3) synthesizing short PANI chains that can undergo gradual erosion [136]. Electrospinning a biocompatible or a biodegradable polymer filled with PANI could produce nanofibers that may be useful in tissue engineering applications. Studies on electrospun PANI blends for tissue engineering include reports on PANI/ chitosan [137], PANI/poly(glycerol-sebacate) (PGS) [138], PANI/nanohydroxyapatite/gelatin [139], PANI/PLA [140], PANI/PPY [141], and PANI/PCL [142].

Previous research conducted by Li et al. [143] described the use of composite nanofibers of PANI blended with gelatin as a conductive scaffold in the proliferation of myoblast cell tissue culture. Gelatin is a frequently used biomaterial for tissue engineering applications, especially in cardiac tissue engineering $[144,145]$. Gelatin, which is less than $3 \%$ compared to the amount of PANI in total weight, leads to an alteration of the physicochemical properties of the nanofiber composites. The decrement in the diameter of the composite nanofibers was found with the increment of the PANI to gelatin volume ratio. The cells initially displayed different morphologies, depending on the concentration of PANI. The cells cultured on the smaller diameter PANI-gelatin nanofiber blend were more spread out and exhibited a smooth musclelike morphology, similarly to the cells growing on a glass cover surface (control sample). However, cell growth on larger diameter nanofiber substrates presented low density and pseudopodia. Other physicochemical measurements, such as tensile strength, elasticity, conductivity, and electron microscopy analysis, have confirmed the uniformity of the ensued PANI nanofibers.

The preliminary study of PANI nanofibers in tissue engineering [143] was then supported with the research works developed by Ghasemi-Mobarakeh et al. It was explained that the surface modification and functionalization of PANI with different biomolecules or dopants allowed them to be modified with biological sensing elements and to exhibit different signaling pathways required for cellular processes. In this way, significant enhancement in cell proliferation and differentiation was observed through the employment of PANI. Thus, conductive PANI provides an excellent opportunity for the fabrication of highly selective, biocompatible, specific, and stable nanocomposite scaffolds for tissue engineering of different organs [146].

The effect of PANI nanofiber orientation on electrical conductivity has also been reported by Ku et al. [147] who prepared electrospun PANI nanofibers in a PCL blend. Rotor speed was adjusted to $100 \mathrm{rpm}$ for collecting random nanofibers and to $900 \mathrm{rpm}$ for aligned nanofibers. The electrospun PANI/PCL nanofibers with both random and aligned orientation were used as scaffolds for the growth of C2C12 mouse myoblast cells. The diameter of the blended PANI-PCL nanofibers was reduced through the increasing concentration of PANI as a result of the increment in electrical conductivity of the spinning solution.

The seeded cells grown on randomly oriented nanofiber matrixes were observed as having flat and multipolar morphologies. In contrast, the seeded cells grown on aligned nanofibers were found to possess bipolar morphologies. PANI loading significantly affected the number of myotubes formed on the PANI-PCL nanofibers. The number of the myotubes formed increased with increasing PANI concentration. The results suggested that both conductivity and alignment of nanofibers can synergistically stimulate the myogenic differentiation.

The development of effective methods to deliver drugs has attracted wide attention, primarily in the fields of tumor therapy and tissue engineering. A controlled drug delivery system can release quantitatively drugs at a specific time and position, especially the stimuli-response drug delivery systems [148-150]. Conducting polymers exhibit a reversible electrochemical response; they will contract upon reduction and expand after oxidation. This feature permits the controlled release of various kinds of drugs [151,152]. Electrospun conducting polymer nanofibers provide high surface area, which can be easily loaded with drugs. It has been shown that the release of dexamethasone from electrospun PEDOT/PGA nanofibers can be controlled by using electrical stimulation, which is due to the contraction and expansion of the PEDOT [153]. Up to date, there have been a few works reported focusing on electrospun PANI blends for controlled drug release [154].

6.2. Sensors. PANI nanofibers are a promising candidate for designing sensors since their conductivity is highly sensitive to chemical vapors due to their high surface area [155]. Synthetically prepared PANI nanofibers $[156,157]$ and PANI 
nanocomposites with carbon nanotubes (CNTs) [158], $\mathrm{WO}_{3}$ [159], and $\mathrm{TiO}_{2}$ [160] have been investigated for gas sensing applications towards humidity and vapors and as biosensor devices, thus making use of the conductivity changes upon oxidation or reduction [161]. This specific feature was targeted to amplify signals transduced by the electrochemical reactions [162-164].

Electrospun PANI nanofibers and their polymeric blends have recently captured the researchers' interest due to their ease of processing, excellent detection, and uniform nanofiber formation, compared to other chemical or template routes. Several types of electrospun PANI nanofiber based sensors have been developed lately. Recent reports include the electrospun nanofiber of graphene/PANI/PS for the detection of $\mathrm{Pb}$ and $\mathrm{Cd}$ ions [165], $\mathrm{PANI} / \mathrm{SnO}_{2}$ for $\mathrm{H}_{2}$ gas sensing [166], multiple detections using PANI/PCL [167], PANI/polyamide 66 for colorimetric sensing [168], PANI/poly(ethylene oxide) gas sensor [169], PANI/ZnO for chemiresistor sensor [170], PANI/PLA to sense alcohol vapors of increasing molecular size [171], PANI/polyvinylidene fluoride as strain sensor [172], high sensitivity gas sensors based on PANI/PMMA [173], PANI/fibroin microfibrous mat as reactive sensor [174], and $\mathrm{PANI} /$ polyamide $6 / \mathrm{TiO}_{2}$ nanofibers for ammonia sensor $[175,176]$.

The humidity response of the PANI nanofiber sensors was determined by measuring their impedance at different humidity levels at room temperature. According to Lin et al. [177], the rheology of PANI nanofibers presenting the formation of some beads exhibited improved adhesion to the electrode receptor and better electrical contact due to the high surface area, which in turn enhanced their sensing properties, compared with the nanofibers without beads. The blending of PEO into PANI greatly modified the hydrophilicity of the PANI-PEO nanofibers, and their humidity response. The PANI-PEO nanofiber based sensors revealed much higher sensitivity than their film counterparts with the impedance increasing by three orders of magnitude from $20 \%$ to $90 \%$ RH. Humidity sensors based on PANI nanofibers with some beads and a small content of PEO revealed high sensitivity, fast response, and small hysteresis [177].

Lin et al. [178], on the other hand, developed humidity sensors of surface acoustic wave (SAW) based on electrospun core-sheath structured polyaniline/poly(vinyl butyral) PANI/PVB blended nanofibers. It was reported that nanostructured PANI exhibited an excellent chemical sensitive response, with higher sensitivity and faster response compared to those of bulk PANI. As it was not possible to prepare nanostructured PANI directly from its solution, it was blended with PVB. The results indicated that PVB was suitable to be coelectrospun with PANI to form a core-sheath structure.

It was demonstrated that high performance SAW humidity sensors could be successfully fabricated based on electrospun core-sheath PANI-PVB composite nanofibers. The composite nanofiber SAW sensor revealed very high sensitivity of $75 \mathrm{kHz} / \% \mathrm{RH}$ over a wide humidity range, good sensing linearity, and very fast response in both humidification and desiccation processes. Moreover, PANI-PVB nanofiber based sensors could be used to detect humidity as low as $0.5 \% \mathrm{RH}$ with good sensitivity. The high surface-to-volume ratio and the improved mass load, electroacoustic load, and viscoelastic load of the core-sheath structured composite nanofibers of PANI blended with PVB were responsible for the attractive humidity sensing properties of the SAW sensors [178].

It is known that the detection stability is an important parameter for a sensor. Doped PANI generally shows unsatisfying stability in the open air, leading to a short lifetime of PANI based sensors. However, the PANI/PVB nanofiber sensor showed good stability, owing to the rheology of the electrospun composite nanofibers, which exhibited a coresheath structure, with PANI inner core, covered by PVB outer sheath, instead of being directly exposed to the open air. The chemically stable and relatively hydrophobic PVB could effectively protect the PANI core. The investigation on the long-term stability of the SAW sensor based on PANIPVB nanofibers found that the sensor maintained its high sensitivity after storing for more than seven months [178].

Electrospun PANI nanofibers doped with palladium nanoparticles have been demonstrated efficient for hydrogen sensing application in the fabrication of a chemiresistor [179]. The nanofibers were deposited as single nanofibers across two gold electrodes by means of near-field electrospinning without using the conventional lithography process. These palladium nanoparticle based chemiresistors recorded $1.8 \%$ resistance change in the environment with $0.3 \%$ hydrogen concentration. These nanofiber sensors are flexible, have good reversibility, and are potentially versatile by doping with different particles.

Further, the study of Zhang and Rutledge reported the fabrication of electrospun fibers of PANI and poly(3,4-ethylenedioxythiophene) (PEDOT), blended with poly(ethylene oxide) (PEO) and poly(methyl methacrylate) (PMMA) over a range of compositions, using template synthesis. Fibers of neat PANI doped with camphor sulfonic acid (CSA) were successfully fabricated for the first time by coaxial electrospinning and subsequent removal of the PMMA shell by dissolution. This allowed for the electrospun PANI/CSA fibers to be tested for electrical performance and its enhancement, as well as for gas sensing application. The conductivities of the PANI-blend fibers were found to increase exponentially with the weight percent of doped PANI in the fibers, to as high as $50 \pm 30 \mathrm{Scm}^{-1}$ for the as-electrospun fibers of $100 \%$ PANI/CSA. The conductivity of the neat doped PANI fibers was found to increase to $130 \pm 40 \mathrm{Scm}^{-1}$ with increasing molecular orientation, achieved through solid state drawing [100].

The experimental results thus support those described previously; that is, enhanced molecular alignment within electrospun fibers, during both the electrospinning process and subsequent posttreatment, contributes positively to increasing the electrical conductivity of conductive polymers. Neat PANI fibers with different levels of doping were also fabricated by coaxial electrospinning and subsequent removal of the shell by dissolution and shown to exhibit a large range of fiber electrical conductivities, which increased exponentially with the increasing ratio of dopant to PANI. These fibers were found to be very effective in nanoscale chemiresistive 
sensors for both ammonia and nitrogen dioxide gases, thanks to this large range of available electrical conductivities. Both sensitivity and response times were shown to be excellent, with response ratios up to 58 for doped PANI sensing of ammonia and up to more than 105 for nitrogen dioxide sensing by undoped PANI fibers. The characteristic times for the gas sensing were shown to be on the order of 1 to $2 \mathrm{~min}$. Using a model that accounted for the effects of intrinsic fiber conductivity (including both composition and molecular orientation), mat porosity, and fiber orientation distribution within the mat, calculated mat conductivities were obtained in quantitative agreement with the mat conductivities measured experimentally [100]. Electrospun PANI/PS nanofiber showed excellent detection of $\mathrm{H}_{2} \mathrm{O}_{2}$ compared to that of its corresponding thin film form. The $\mathrm{H}_{2} \mathrm{O}_{2}$ is useful because it is often a product of enzymatic reactions. It is generated in the reaction between glucose and oxygen in the presence of glucose oxidase (GOX). The large surface area promotes more GOX immobilization than the film sensor [176].

6.3. Supercapacitors. The increasing energy need of the world population has urged for new discoveries and manipulation of renewable energy sources. However, it is crucial to find ways to store energy in order to keep getting sufficient supply and to secure energy distribution. For this reason, the supercapacitor has been a phenomenal discovery with its low internal resistance, fast charge and discharge, high power density, high energy density, and long life cycle, compared to conventional capacitors and batteries [180-184]. The key technology that underlies its performance and has turned it into a research hotspot is the electrode material. Electrode materials mainly include carbon material, metal oxide, and conducting polymers. Among conductive polymers, PANI has become the preferred material due to its high capacitance, desirable chemical stability, and multiple intrinsic redox states [185].

Given all these excellent advantages of PANI, it has become a strong competitor as a material for building powerful supercapacitors. Recently, researchers have recognized this potential of electrospun PANI through their studies on electrospun PANI nanofiber web electrodes [186], threedimensional porous PANI/polyacrylonitrile core-shell [187], sandwiched symmetric supercapacitor consisting of flexible PANI/carbonized polyimide [188], PANI/GO/PVDF [189], hollow-structured PANI [91], and PANI/vanadium pentoxide as asymmetric supercapacitor [190].

Chaudhari and his coworkers [186] initiated a study on applying PANI nanofiber webs as electrode materials for supercapacitors. Since the direct processing of PANI by electrospinning is a difficult task, they proposed the fabrication of high aspect ratio PANI $(>50)$ by electrospinning a polymeric blend of PANI with polyethylene oxide (PEO). The fabrication of electrodes took $75 \mathrm{wt} \%$ of PANI, $15 \mathrm{wt} \%$ of carbon black, $10 \mathrm{wt} \%$ of polyvinylidene fluoride, and a few drops of $N$-methylpyrrolidinone to form a uniform slurry. The resulting slurry was then used to coat a graphite substrate, which served as current collector.

The performance of electrospun nanofibers produced in aqueous electrolyte $\left(1 \mathrm{M} \mathrm{H}_{2} \mathrm{SO}_{4}\right)$ and organic $\left(1 \mathrm{M} \mathrm{LiClO}_{4}\right.$ in propylene carbonate (PC)) electrolyte is compared with that of PANI powder. The specific capacitance of a PANI nanofiber web in $1 \mathrm{M} \mathrm{H}_{2} \mathrm{SO}_{4}$ is $267 \mathrm{~F}^{-1}{ }^{-1}$ at a current density of $0.35 \mathrm{~A} \cdot \mathrm{g}^{-1}$, which is higher compared to that of PANI powder $\left(208 \mathrm{~F} \cdot \mathrm{g}^{-1}\right)$. PANI nanofiber webs demonstrate superior stable performance compared to that of PANI powder and deliver a specific capacitance of $230 \mathrm{~F} \cdot \mathrm{g}^{-1}$ at the 1000th cycle. Over $86 \%$ of its original capacitance is retained after 1000 cycles; meanwhile, the capacitance retention of the PANI powder is observed to be $48 \%$ after 1000 cycles. This observation indicated that PANI nanofiber webs have better cycle stability than PANI powder. Smaller individual impedance parameters in the case of PANI nanofiber webs were also observed, indicating the availability of electroactive sites needed for charge transfer reaction and counterion diffusion. Given these characteristics demonstrated by PANI nanofibers synthesized through electrospinning, it was found to be a suitable candidate for being used as electrode material in high performance supercapacitors [186].

6.4. Solar Cells. Dye-sensitized solar cells (DSCs) have turned out to be the centre of interest in nowaday's lowcarbon economy as a solution to the energy requirements $[191,192]$. They have been recognized as a potential alternative to silicon based solar cells due to their simple fabrication procedures, high catalytic performance, and cheap counter electrodes [193]. Counter electrodes of DSCs function as collecting electrons, catalyzing the redox couple regeneration. These are usually made of Pt films, but despite their good performance, $\mathrm{Pt}$ based solar cells need high temperature of operation and complex facilities, which limits their application [194-196]. For these reasons, recent decades have witnessed the development of PANI usage in this area in order to enhance cell stability, reduce fabrication cost, and simplify the preparation techniques, compared to previously made solar cells [197]. Duan et al. also proved that a solid PANI electrolyte has the ability of catalyzing tri-iodide species, shortening the charge diffusion path length, and recovering dye molecules at the anode/electrolyte interface [197]. The focus of the research on utilizing PANI as a component in DSCs has widened by the effort of certain research teams in studying electrospun PANI nanocomposites. However, this is still an emerging topic of research; therefore the studies dedicated to it seem scarce, regardless of the potential shown. An investigation on electrospun PLA/PANI nanofibers for rigid and flexible DSCs [198] and another on PANI/MWCNT counter electrodes [199] are among the few studies conducted.

A simple electrospinning method was developed [198] to directly deposit conductive 10-camphorsulfonic acid (CSA) doped PANI blended with polylactic acid (PLA) composite films (PANI/CSA-PLA) on flexible indium tin oxide-coated polyethylenenaphthalate (PEN) and rigid fluorine doped tin oxide (FTO) substrates. The result revealed that the catalytic performance of the PANI/CSA-PLA film was higher than that of the PANI-PLA film, which was ascribed to the contribution of the high surface area of PANI/CSA-PLA nanofibers and relatively high electrical conductivity of the PANI/CSA-PLA 
film. The preparation process of the PANI/CSA-PLA counter electrodes suggested in this experiment was simple and cost-efficient, and thus feasible for large-scale and flexible application of DSCs.

The flexible and rigid DSCs demonstrate high photovoltaic efficiency, close to that of sputtered Pt based DSCs. It was found that the flexible DSC based on the PANI/CSAPLA counter electrode can exhibit a conversion efficiency of $3.1 \%$, while the PANI/CSA-PLA film on FTO glass as counter electrode exhibits a reasonably high photoelectrical conversion efficiency of $5.30 \%$. Although these results are a little lower than those for the sputtered Pt based DSC (flexible $=4.39 \%$, rigid $=6.51 \%$ ), they demonstrate that PANI can be used as counter electrode material for DSCs, providing efficiency values comparable to those of the Pt based device [198].

\section{Conclusions}

(1) Electrospinning is a simple, versatile, and rapid technology advancement, which can generate nonwoven nanofibers with high surface area to volume ratio and tunable porosity and can be designed into various fiber forms. Due to these interesting properties, the electrospinning process seems to be a promising method for various applications.

(2) The polymer solution, the needle, and the collector used in electrospinning have significant effects on the fiber morphology; thus by the manipulation of these parameters one can get nanofibers with desired properties for targeted applications.

(3) The introduction of polymer blends and fillers into PANI solution is an alternative for the electrospinning of PANI, apart from template coating, which can enhance the processability of PANI.

(4) Electrospun PANI nanofiber blends and composites are being tested to develop new smart materials for a variety of applications, such as tissue engineering scaffolds, sensors, supercapacitors, and solar cells. The materials are designed to respond by changing the shape and size of the cells and have demonstrated high sensitivity of detection toward specific environments.

(5) In general, electrospun PANI nanofibers show an excellent potential for future applications, particularly in biomedical devices and sensors. However, the studies on biocompatibility for long-term use are still inadequately carried out and are rather limited. It is important that the toxicology profile of the electrospun PANI nanofibers and their health impacts be evaluated before the actual industrial production begins.

(6) The core-sheath structure of PANI hybrids with other polymers has improved the usability of PANI. The limitation of PANI stability over long periods of time due to dopant leaching, which may have a negative impact on the ambient environment, should be thus resolved.
(7) In addition to issues on extended response/recovery capabilities, the viability of PANI nanofibers is also being held back by poor mechanical properties as PANI tends to be a brittle material with poor ductility. Although films of PANI nanofibers can be deposited onto many substrates, it is not possible to produce PANI nanofiber films, which are mechanically robust enough to be free-standing. A new method towards free-standing PANI nanofibers by improving the mechanical properties is another way to enhance the usability of PANI nanofibers for a wide range of future biomedical, energy, and biotechnology applications.

\section{Conflict of Interests}

The authors declare that there is no conflict of interests regarding the publication of this paper.

\section{Acknowledgments}

The authors would to express their gratitude to Universiti Teknologi Malaysia for the Potential Academic Staff (PAS) scheme, IJN-UTM Cardiovascular Engineering Centre, Faculty of Bioscience and Medical Engineering, and also Department of Chemistry, Faculty of Science, Universiti Teknologi Malaysia.

\section{References}

[1] V. M. Agranovich, Y. N. Gartstein, and M. Litinskaya, "Hybrid resonant organic-inorganic nanostructures for optoelectronic applications," Chemical Reviews, vol. 111, no. 9, pp. 5179-5214, 2011.

[2] R. Li, W. Hu, Y. Liu, and D. Zhu, "Micro- and nanocrystals of organic semiconductors," Accounts of Chemical Research, vol. 43, no. 4, pp. 529-540, 2010.

[3] H. Qu, S. Wei, and Z. Guo, "Coaxial electrospun nanostructures and their applications," Journal of Materials Chemistry A, vol. 1, no. 38, pp. 11513-11528, 2013.

[4] L. Xia, Z. Wei, and M. Wan, "Conducting polymer nanostructures and their application in biosensors," Journal of Colloid and Interface Science, vol. 341, no. 1, pp. 1-11, 2010.

[5] I. Y. Goryacheva, E. S. Speranskaya, V. V. Goftman, D. Tang, and S. De Saeger, "Synthesis and bioanalytical applications of nanostructures multiloaded with quantum dots," TrAC-Trends in Analytical Chemistry, vol. 66, pp. 53-62, 2015.

[6] T. M. Hsieh, A. C. A. Wan, and J. Y. Ying, "Applications of fabricated micro- and nanostructures in biomedicine," MRS Bulletin, vol. 36, no. 12, pp. 990-997, 2011.

[7] H. Liu, X. Ding, G. Zhou, P. Li, X. Wei, and Y. Fan, "Electrospinning of nanofibers for tissue engineering applications," Journal of Nanomaterials, vol. 2013, Article ID 495708, 11 pages, 2013.

[8] A. G. MacDiarmid, “"Synthetic metals”: A novel role for organic polymers (Nobel lecture)," Angewandte Chemie - International Edition, vol. 40, no. 14, pp. 2581-2590, 2001.

[9] G. A. Snook, P. Kao, and A. S. Best, "Conducting-polymerbased supercapacitor devices and electrodes," Journal of Power Sources, vol. 196, no. 1, pp. 1-12, 2011. 
[10] S. I. A. Razak, N. F. A. Sharif, and N. H. M. Nayan, "Electrically conductive paper of polyaniline modified pineapple leaf fiber," Fibers and Polymers, vol. 15, no. 6, pp. 1107-1111, 2014.

[11] Y.-Z. Long, M.-M. Li, C. Gu et al., "Recent advances in synthesis, physical properties and applications of conducting polymer nanotubes and nanofibers," Progress in Polymer Science, vol. 36, no. 10, pp. 1415-1442, 2011.

[12] J. Zeleny, "Instability of electrified liquid surfaces," Physical Review, vol. 10, no. 1, pp. 1-6, 1917.

[13] A. Frenot and I. S. Chronakis, "Polymer nanofibers assembled by electrospinning," Current Opinion in Colloid \& Interface Science, vol. 8, no. 1-2, pp. 64-75, 2003.

[14] J. Dias, F. Antunes, and P. Bartolo, "Influence of the rheological behavior in electrospun PCL nanofibers production for tissue engineering applications," Chemical Engineering Transactions, vol. 32, pp. 1015-1020, 2013.

[15] X. Y. Wang, C. Drew, S. H. Lee, K. J. Senecal, J. Kumar, and L. A. Sarnuelson, "Electrospunna nofibrous membranes for highly sensitive optical sensors," Nano Letters, vol. 2, pp. 1273-1275, 2002.

[16] A. R. Phani, R. M. T. De Britto, and S. Srinivasan, "Polyaniline nanofibers obtained by electrospin process for hydrogen storage applications," International Journal of Environmental Research and Development, vol. 4, pp. 375-386, 2014.

[17] F. K. Ko and H. Yang, "Functional nanofibers: enabling material for the net generations smart textiles," Journal of Fiber Bioengineering and Informatics, vol. 1, no. 2, pp. 81-92, 2008.

[18] T. Jaroszczyk, S. Petrik, and K. Donahue, "The role of nanofiber filter media in motor vehicle air filtration," in Proceedings of the 4th Biennial Conference on Emissions Solutions in Transportation, AFS, Ann Arbor, Mich, USA, October 2009.

[19] N. Khan, "Applications of electrospun nanofibers in the biomedical field," Studies by Undergraduate Researchers at Guelph, vol. 5, pp. 63-73, 2012.

[20] Z.-M. Huang, Y.-Z. Zhang, M. Kotaki, and S. Ramakrishna, "A review on polymer nanofibers by electrospinning and their applications in nanocomposites," Composites Science and Technology, vol. 63, no. 15, pp. 2223-2253, 2003.

[21] J. Zeleny, "The electrical discharge from liquid points, and a hydrostatic method of measuring the electric intensity at their surfaces," Physical Review, vol. 3, no. 2, pp. 69-91, 1914.

[22] A. Formhals, "Process and apparatus for preparing artificial threads," U.S. Patent no. 1975704, 1934.

[23] C. L. Norton, "Method and apparatus for producing fibrous or filamentary material," U.S. Patent No. 2408651, 1936.

[24] G. S. Taylor, "Electrically driven jets," Proceedings of Royal Society of London A, vol. 31, pp. 353-475, 1969.

[25] P. Bhattarai, K. B. Thapa, R. B. Basnet, and S. Sharma, "Electrospinning: how to produce nanofibers using most inexpensive technique? An insight into the real challenges of electrospinning such nanofibers and its application areas," International Journal of Biomedical and Advance Research, vol. 5, no. 9, pp. 401-405, 2014.

[26] S. Ramakrishna, K. Fujihara, W.-E. Teo, T. Yong, Z. Ma, and R. Ramaseshan, "Electrospun nanofibers: solving global issues," Materials Today, vol. 9, no. 3, pp. 40-50, 2006.

[27] X. Fang and D. H. Reneker, "DNA fibers by electrospinning," Journal of Macromolecular Science-Physics, vol. 36, no. 2, pp. 169-173, 1997.

[28] F. Fadil, N. A. Buang, M. P. Prabhakaran, and S. Ramakrishna, "The diameter size reduction of composited poly $\mathcal{\varepsilon}$ caprolactone-co-lactide (PLCL) nanofibers using multi-walled carbon nanotubes (MWCNTs) as the nanofiller," Digest Journal of Nanomaterials and Biostructures, vol. 10, no. 1, article A2, pp. 11-17, 2015.

[29] J. Doshi and D. H. Reneker, "Electrospinning process and applications of electrospun fibers," Journal of Electrostatics, vol. 35, no. 2-3, pp. 151-160, 1995.

[30] H. Fong, I. Chun, and D. H. Reneker, "Beaded nanofibers formed during electrospinning," Polymer, vol. 40, no. 16, pp. 4585-4592, 1999.

[31] C. J. Luo, M. Nangrejo, and M. Edirisinghe, "A novel method of selecting solvents for polymer electrospinning," Polymer, vol. 51, no. 7, pp. 1654-1662, 2010.

[32] S. H. Bahrami and A. Gholipour Kanani, "Effect of changing solvents on poly( $\varepsilon$-Caprolactone) nanofibrous webs morphology," Journal of Nanomaterials, vol. 2011, Article ID 724153, 10 pages, 2011.

[33] F. Yalcinkaya, B. Yalcinkaya, and O. Jirsak, "Influence of salts on electrospinning of aqueous and nonaqueous polymer solutions," Journal of Nanomaterials, vol. 2015, Article ID 134251, 12 pages, 2015.

[34] C. Migliaresi, G. A. Ruffo, F. Z. Volpato, and D. Zeni, Advanced Electrospinning Setups and Special Fibre and Mesh Morphologies Advanced Electrospinning Setups and Special Fibre and Mesh Morphologies, Smithers Rapra, Akron, Ohio, USA, 2012.

[35] W. Bonani, D. Maniglio, A. Motta, W. Tan, and C. Migliaresi, "Biomedical gradient in micropatterned nanofibrous scaffold for spatiotemporal release," Journal of Biomedical Materials Research Part B: Applied Biomaterials, vol. 96, no. 2, pp. 276286, 2011.

[36] T. Lin, H. Wang, and X. Wang, "Self-crimping bicomponent nanofibers electrospun from polyacrylonitrile and elastomeric polyurethane," Advanced Materials, vol. 17, no. 22, pp. 26992703, 2005.

[37] P. Gupta and G. L. Wilkes, "Some investigations on the fiber formation by utilizing a side-by-side bicomponent electrospinning approach," Polymer, vol. 44, no. 20, pp. 6353-6359, 2003.

[38] M. K. Leach, Z.-Q. Feng, S. J. Tuck, and J. M. Corey, “Electrospinning fundamentals: optimizing solution and apparatus parameters," Journal of Visualized Experiments, vol. 47, pp. 1-4, 2011.

[39] Y. Wu, Z. Dong, S. Wilson, and R. L. Clark, “Template-assisted assembly of electrospun fibers," Polymer, vol. 51, no. 14, pp. 3244-3248, 2010.

[40] C. Vaquette and J. J. Cooper-White, "Increasing electrospun scaffold pore size with tailored collectors for improved cell penetration," Acta Biomaterialia, vol. 7, no. 6, pp. 2544-2557, 2011.

[41] D. Zhang and J. Chang, "Electrospinning of three-dimensional nanofibrous tubes with controllable architectures," Nano Letters, vol. 8, no. 10, pp. 3283-3287, 2008.

[42] T. Kongkhlang, K. Tashiro, M. Kotaki, and S. Chirachanchai, "Electrospinning as a new technique to control the crystal morphology and molecular orientation of polyoxymethylene nanofibers," Journal of the American Chemical Society, vol. 130, no. 46, pp. 15460-15466, 2008.

[43] B. Lu, Y. Wang, Y. Liu et al., "Superhigh-throughput needleless electrospinning using a rotary cone as spinneret," Small, vol. 6, no. 15, pp. 1612-1616, 2010.

[44] S. Tang, Y. Zeng, and X. Wang, "Splashing needleless electrospinning of nanofibers," Polymer Engineering and Science, vol. 50, no. 11, pp. 2252-2257, 2010. 
[45] H. Niu, X. Wang, and T. Lin, "Upward needleless electrospinning of nanofibers," Journal of Engineered Fibers and Fabrics, vol. 7, no. 3, pp. 17-22, 2012.

[46] A. J. Heeger, "Semiconducting polymers: the third generation," Chemical Society Reviews, vol. 39, no. 7, pp. 2354-2371, 2010.

[47] X. F. Lu, W. J. Zhang, C. Wang, T.-C. Wen, and Y. Wei, “Onedimensional conducting polymer nanocomposites: synthesis, properties and applications," Progress in Polymer Science, vol. 36, no. 5, pp. 671-712, 2011.

[48] J. Stejskal, I. Sapurina, and M. Trchová, "Polyaniline nanostructures and the role of aniline oligomers in their formation," Progress in Polymer Science, vol. 35, no. 12, pp. 1420-1481, 2010.

[49] X. Ding, D. Han, Z. Wang, X. Xu, L. Niu, and Q. Zhang, "Micelle-assisted synthesis of polyaniline/magnetite nanorods by in situ self-assembly process," Journal of Colloid and Interface Science, vol. 320, no. 1, pp. 341-345, 2008.

[50] B.-J. Kim, S.-G. Oh, M.-G. Han, and S.-S. Im, "Preparation of polyaniline nanoparticles in micellar solutions as polymerization medium," Langmuir, vol. 16, no. 14, pp. 5841-5845, 2000.

[51] Y. Guo and Y. Zhou, "Polyaniline nanofibers fabricated by electrochemical polymerization: a mechanistic study," European Polymer Journal, vol. 43, no. 6, pp. 2292-2297, 2007.

[52] L. I. Dan, J. Huang, and R. B. Kaner, "Polyaniline nanofibers: a unique polymer nanostructure for versatile applications," Accounts of Chemical Research, vol. 42, no. 1, pp. 135-145, 2009.

[53] S. Virji, J. Huang, R. B. Kaner, and B. H. Weiller, "Polyaniline nanofiber gas sensors: examination of response mechanisms," Nano Letters, vol. 4, no. 3, pp. 491-496, 2004.

[54] J. Wang, S. Chan, R. R. Carlson et al., "Electrochemically fabricated polyaniline nanoframework electrode junctions that function as resistive sensors," Nano Letters, vol. 4, no. 9, pp. 1693-1697, 2004.

[55] J. Huang, "Syntheses and applications of conducting polymer polyaniline nanofibers," Pure and Applied Chemistry, vol. 78, no. 1, pp. 15-27, 2006.

[56] D. Li and R. B. Kaner, "Processable stabilizer-free polyaniline nanofiber aqueous colloids," Chemical Communications, no. 26, pp. 3286-3288, 2005.

[57] N. Densakulprasert, L. Wannatong, D. Chotpattananont, P. Hiamtup, A. Sirivat, and J. Schwank, "Electrical conductivity of polyaniline/zeolite composites and synergetic interaction with CO," Materials Science and Engineering B: Solid-State Materials for Advanced Technology, vol. 117, no. 3, pp. 276-282, 2005.

[58] J. Gao, J.-M. Sansiñena, and H.-L. Wang, "Chemical vapor driven polyaniline sensor/actuators," Synthetic Metals, vol. 135136, pp. 809-810, 2003.

[59] K. Ghanbari, M. F. Mousavi, M. Shamsipur, and H. Karami, "Synthesis of polyaniline/graphite composite as a cathode of Zn-polyaniline rechargeable battery," Journal of Power Sources, vol. 170, no. 2, pp. 513-519, 2007.

[60] J. Huang and R. B. Kaner, "A general chemical route to polyaniline nanofibers," Journal of the American Chemical Society, vol. 126, no. 3, pp. 851-855, 2004.

[61] T. S. Najim and A. J. Salim, "Polyaniline nanofibers and nanocomposites: preparation, characterization, and application for $\mathrm{Cr}(\mathrm{VI})$ and phosphate ions removal from aqueous solution," Arabian Journal of Chemistry, 2014.

[62] R. A. Green, S. Baek, L. A. Poole-Warren, and P. J. Martens, "Conducting polymer-hydrogels for medical electrode applications," Science and Technology of Advanced Materials, vol. 11, no. 1, Article ID 014107, 2010.
[63] S. I. A. Razak, N. F. A. Sharif, and I. I. Muhamad, "Polyanilinecoated halloysite nanotubes: effect of para-hydroxybenzene sulfonic acid doping," Composite Interfaces, vol. 21, no. 8, pp. 715-722, 2014.

[64] S. I. A. Razak, W. A. W. A. Rahman, and M. Y. Yahya, "Electrically conductive nanocomposites of epoxy/polyaniline nanowires doped with formic acid: effect of loading on the conduction and mechanical properties," Nano, vol. 7, no. 5, Article ID 1250039, 2012.

[65] C. R. Martin, "Nanomaterials-a membrane-based synthetic approach," Science, vol. 266, no. 5193, pp. 1961-1966, 1994.

[66] X. Sun, S. Dong, and E. Wang, "Formation of o-phenylenediamine oligomers and their self-assembly into onedimensional structures in aqueous medium," Macromolecular Rapid Communications, vol. 26, no. 18, pp. 1504-1508, 2005.

[67] G. Chang, Y. Luo, W. Lu et al., "Ag nanoparticles decorated polyaniline nanofibers: synthesis, characterization, and applications toward catalytic reduction of 4-nitrophenol and electrochemical detection of $\mathrm{H}_{2} \mathrm{O}_{2}$ and glucose," Catalysis Science and Technology, vol. 2, no. 4, pp. 800-806, 2012.

[68] M. X. Wan, "A template-free method towards conducting polymer nanostructures," Advanced Materials, vol. 20, no. 15, pp. 2926-2932, 2008.

[69] M. X. Wan, J. Huang, and Y. Q. Shen, "Microtubes of conducting polymers," Synthetic Metals, vol. 101, no. 1, pp. 708-711, 1999.

[70] L. J. Zhang, Y. Z. Long, Z. J. Chen, and M. X. Wan, “The effect of hydrogen bonding on self-assembled polyaniline nanostructures," Advanced Functional Materials, vol. 14, no. 7, pp. 693-698, 2004.

[71] H. J. Ding, J. Y. Shen, M. X. Wan, and Z. J. Chen, "Formation mechanism of polyaniline nanotubes by a simplified templatefree method," Macromolecular Chemistry and Physics, vol. 209, no. 8, pp. 864-871, 2008.

[72] H. J. Ding, M. X. Wan, and Y. Wei, "Controlling the diameter of polyaniline nanofibers by adjusting the oxidant redox potential," Advanced Materials, vol. 19, no. 3, pp. 465-469, 2007.

[73] H. Ding, Y. Long, J. Shen, and M. Wan, " $\mathrm{Fe}_{2}\left(\mathrm{SO}_{4}\right)_{3}$ as a binary oxidant and dopant to thin polyaniline nanowires with high conductivity," Journal of Physical Chemistry B, vol. 114, no. 1, pp. 115-119, 2010

[74] Y. Yan, K. Deng, Z. Yu, and Z. X. Wei, "Tuning the supramolecular chirality of polyaniline by methyl substitution," Angewandte Chemie: International Edition in English, vol. 48, no. 11, pp. 2003-2006, 2009.

[75] L. J. Zhang and M. X. Wan, "Self-assembly of polyanilinefrom nanotubes to hollow microspheres," Advanced Functional Materials, vol. 13, no. 10, pp. 815-820, 2003.

[76] L. J. Zhang, M. X. Wan, and Y. Wei, "Hollow polyaniline microspheres with conductive and fluorescent function," Macromolecular Rapid Communications, vol. 27, no. 11, pp. 888-893, 2006.

[77] Z. Zhang, Z. Wei, L. Zhang, and M. Wan, "Polyaniline nanotubes and their dendrites doped with different naphthalene sulfonic acids," Acta Materialia, vol. 53, no. 5, pp. 1373-1379, 2005.

[78] A. Greiner and J. H. Wendorff, "Functional self-assembled nanofibers by electrospinning," Advances in Polymer Science, vol. 219, no. 1, pp. 107-171, 2008.

[79] J.-Y. Kim, J.-H. Lee, and S.-J. Kwon, "The manufacture and properties of polyaniline nano-films prepared through vaporphase polymerization," Synthetic Metals, vol. 157, no. 8-9, pp. 336-342, 2007. 
[80] T.-S. Jun, C.-K. Kim, and Y. S. Kim, "Vapor phase polymerization of polyaniline nanotubes using $\mathrm{Mn}_{3} \mathrm{O}_{4}$ nanofibers as an oxidant," Materials Letters, vol. 133, pp. 17-19, 2014.

[81] J. M. D’Arcy, M. F. El-Kady, P. P. Khine et al., "Vapor-phase polymerization of nanofibrillar poly(3,4-ethylenedioxythiophene) for supercapacitors," ACS Nano, vol. 8, no. 2, pp. 1500-1510, 2014.

[82] J.-E. Österholm, Y. Cao, F. Klavetter, and P. Smith, "Emulsion polymerization of aniline," Polymer, vol. 35, no. 13, pp. 29022906, 1994.

[83] P. J. Kinlen, J. Liu, Y. Ding, C. R. Graham, and E. E. Remsen, "Emulsion polymerization process for organically soluble and electrically conducting polyaniline," Macromolecules, vol. 31, no. 6, pp. 1735-1744, 1998.

[84] S. Palaniappan and A. John, "Polyaniline materials by emulsion polymerization pathway," Progress in Polymer Science, vol. 33, no. 7, pp. 732-758, 2008.

[85] X. Jing, Y. Wang, D. Wu, and J. Qiang, "Sonochemical synthesis of polyaniline nanofibers," Ultrasonics Sonochemistry, vol. 14, no. 1, pp. 75-80, 2007.

[86] B. A. Bhanvase, N. S. Darda, N. C. Veerkar, A. S. Shende, S. R. Satpute, and S. H. Sonawane, "Ultrasound assisted synthesis of $\mathrm{PANI} / \mathrm{ZnMoO} \mathrm{M}_{4}$ nanocomposite for simultaneous improvement in anticorrosion, physico-chemical properties and its application in gas sensing," Ultrasonics Sonochemistry, vol. 24, pp. 8797, 2015.

[87] E. J. V. Rani, K. Karthika, N. Saranya, and R. Padmavathy, “The influence of temperature and sonication on the preparation of doped polymeric nano particles," International Journal of ChemTech Research, vol. 6, no. 13, pp. 5168-5173, 2014.

[88] D. D. Sawall, R. M. Villahermosa, R. A. Lipeles, and A. R. Hopkins, "Interfacial polymerization of polyaniline nanofibers grafted to Au surfaces," Chemistry of Materials, vol. 16, no. 9, pp. 1606-1608, 2004.

[89] X. B. Yan, Z. J. Han, Y. Yang, and B. K. Tay, " $\mathrm{NO}_{2}$ gas sensing with polyaniline nanofibers synthesized by a facile aqueous/organic interfacial polymerization," Sensors and Actuators B: Chemical, vol. 123, no. 1, pp. 107-113, 2007.

[90] W. Yang, Z. Gao, N. Song, Y. Zhang, Y. Yang, and J. Wang, "Synthesis of hollow polyaniline nano-capsules and their supercapacitor application," Journal of Power Sources, vol. 272, pp. 915-921, 2014.

[91] P. Frontera, C. Busacca, P. Antonucci et al., "Polyaniline nanofibers: towards pure electrospun PANI," AIP Conference Proceedings, vol. 1459, pp. 253-255, 2012.

[92] J. H. Yu, S. V. Fridrikh, and G. C. Rutledge, "The role of elasticity in the formation of electrospun fibers," Polymer, vol. 47, no. 13, pp. 4789-4797, 2006.

[93] H. J. Yu, S. V. Fridrikh, and G. C. Rutledge, "Production of submicrometer diameter fibers by two-fluid electrospiiming," Advanced Materials, vol. 16, no. 17, pp. 1562-1566, 2004.

[94] N. J. Pinto, A. T. Johnson Jr., A. G. MacDiarmid et al., "Electrospun polyaniline/polyethylene oxide nanofiber fieldeffect transistor," Applied Physics Letters, vol. 83, no. 20, pp. 4244-4246, 2003.

[95] J. R. Cárdenas, M. G. O. de França, E. A. de Vasconcelos, W. M. de Azevedo, and E. F. da Silva Jr., "Growth of sub-micron fibres of pure polyaniline using the electrospinning technique," Journal of Physics D: Applied Physics, vol. 40, pp. 1068-1071, 2007.

[96] H. Liu, J. Kameoka, D. A. Czaplewski, and H. G. Craighead, "Polymeric nanowire chemical sensor," Nano Letters, vol. 4, no. 4, pp. 671-675, 2004.
[97] Y. Zhou, M. Freitag, J. Hone et al., "Fabrication and electrical characterization of polyaniline-based nanofibers with diameter below 30 nm," Applied Physics Letters, vol. 83, no. 18, pp. 38003802, 2003.

[98] I. D. Norris, M. M. Shaker, F. K. Ko, and A. G. MacDiarmid, "Electrostatic fabrication of ultrafine conducting fibers: polyaniline/polyethylene oxide blends," Synthetic Metals, vol. 114, no. 2, pp. 109-114, 2000.

[99] A. G. MacDiarmid, W. E. Jones Jr., I. D. Norris et al., "Electrostatically-generated nanofibers of electronic polymers," Synthetic Metals, vol. 119, no. 1-3, pp. 27-30, 2001.

[100] M. F. Shie, W. T. Li, C. F. Dai, and J. M. Yeh, "In vitro biocompatibility of electrospinning polyaniline fibers," IFMBE Proceedings, vol. 25, no. 10, pp. 211-214, 2009.

[101] M. J. Díaz-de León, "Electrospinning nanofibers of polyaniline and polyaniline/(polystyrene and polyethylene oxide) blends," in Proceeding of the National Conference on Undergraduate Research (NCUR '01), Lexington, Kentucky, March 2001.

[102] Y. Chen, C. Li, Z. Hou et al., "Polyaniline electrospinning composite fibers for orthotopic photothermal treatment of tumors in vivo," New Journal of Chemistry, vol. 39, no. 6, pp. 4987-4993, 2015.

[103] R. Fryczkowski, M. Gorczowska, B. Fryczkowska, and J. Janicki, "The effect of solvent on the properties of nanofibres obtained by electrospinning from a mixture of poly(3-hydroxybutyrate) and polyaniline," Synthetic Metals, vol. 166, no. 1, pp. 14-21, 2013.

[104] M. R. Karim, "Fabrication of electrospun aligned nanofibers from conducting polyaniline copolymer/polyvinyl alcohol/ chitosan oligossacaride in aqueous solutions," Synthetic Metals, vol. 178, pp. 34-37, 2013.

[105] Y. Sharma, A. Tiwari, S. Hattori et al., "Fabrication of conducting electrospun nanofibers scaffold for three-dimensional cells culture," International Journal of Biological Macromolecules, vol. 51, no. 4, pp. 627-631, 2012.

[106] P. Zamani, D. Higgins, F. Hassan et al., "Electrospun ironpolyaniline-polyacrylonitrile derived nanofibers as nonprecious oxygen reduction reaction catalysts for PEM fuel cells," Electrochimica Acta, vol. 139, pp. 111-116, 2014.

[107] A. Moayeri and A. Ajji, "Fabrication of polyaniline/ poly(ethylene oxide)/non-covalently functionalized graphene nanofibers via electrospinning," Synthetic Metals, vol. 200, pp. 7-15, 2015.

[108] Z. Sun, E. Zussman, A. L. Yarin, J. H. Wendorff, and A. Greiner, "Compound core-shell polymer nanofibers by coelectrospinning," Advanced Materials, vol. 15, no. 22, pp. 19291932, 2003.

[109] Y. Zhang and G. C. Rutledge, "Electrical conductivity of electrospun polyaniline and polyaniline-blend fibers and mats," Macromolecules, vol. 45, no. 10, pp. 4238-4246, 2012.

[110] A. Sarvi, V. Chimello, A. B. Silva, R. E. S. Bretas, and U. Sundararaj, "Coaxial electrospun nanofibers of poly(vinylidene fluoride)/polyaniline filled with multi-walled carbon nanotubes," Polymer Composites, vol. 35, no. 6, pp. 1198-1203, 2014.

[111] Q. Wu, D. Wu, and Y. Guan, "Polyaniline sheathed electrospun nanofiber bar for in vivo extraction of trace acidic phytohormones in plant tissue," Journal of Chromatography A, vol. 1342, pp. 16-23, 2014.

[112] Q. Wu, D. Wu, and Y. Guan, "In vivo fast equilibrium microextraction by stable and biocompatible nanofiber membrane sandwiched in microfluidic device," Analytical Chemistry, vol. 85, no. 23, pp. 11524-11531, 2013. 
[113] Q. Ma, J. Wang, X. Dong, W. Yu, and G. Liu, "Flexible ribbonshaped coaxial electrical conductive nanocable array endowed with magnetism and photoluminescence," RSC Advances, vol. 5, no. 4, pp. 2523-2530, 2015.

[114] H. Shao, Q. Ma, X. Dong et al., "Novel flexible belt-shaped coaxial microcables with tunable multicolor luminescence, electrical conductivity and magnetism," Physical Chemistry Chemical Physics, vol. 17, no. 34, pp. 21845-21855, 2015.

[115] Y. Liu, Q. Ma, X. Dong, W. Yu, J. Wang, and G. Liu, "A novel strategy to directly fabricate flexible hollow nanofibers with tunable luminescence-electricity-magnetism trifunctionality using one-pot electrospinning," Physical Chemistry Chemical Physics, vol. 17, no. 35, pp. 22977-22984, 2015.

[116] Y. Liu, Q. Ma, M. Yang et al., "Flexible hollow nanofibers: novel one-pot electrospinning construction, structure and tunable luminescence-electricity-magnetism trifunctionality," Chemical Engineering Journal, vol. 284, pp. 831-840, 2016.

[117] Y. E. Miao, W. Fan, D. Chen, and T. Liu, "High-performance supercapacitors based on hollow polyaniline nanofibers by electrospinning," ACS Applied Materials \& Interfaces, vol. 5, no. 10, pp. 4423-4428, 2013.

[118] N. Lv, X. Dong, Q. Ma, J. Wang, W. Yu, and G. Liu, "Parallel spinnerets electrospinning construct and properties of electricalluminescent bifunctional bistrand-aligned nanobundles," Journal of Materials Science, vol. 49, no. 5, pp. 2171-2179, 2014.

[119] K. H. Lee, H. Y. Kim, Y. J. Ryu, K. W. Kim, and S. W. Choi, "Mechanical behavior of electrospun fiber mats of poly(vinyl chloride)/polyurethane polyblends," Journal of Polymer Science Part B: Polymer Physics, vol. 41, no. 11, pp. 1256-1262, 2003.

[120] L. S. Nair, S. Bhattacharyya, J. D. Bender et al., "Fabrication and optimization of methylphenoxy substituted polyphosphazene nanofibers for biomedical applications," Biomacromolecules, vol. 5, no. 6, pp. 2212-2220, 2004.

[121] K. H. Lee, H. Y. Kim, M. S. Khil, Y. M. Ra, and D. R. Lee, "Characterization of nano-structured poly( $\varepsilon$-caprolactone) nonwoven mats via electrospinning," Polymer, vol. 44, no. 4, pp. 1287-1294, 2003.

[122] J. Yao, C. W. M. Bastiaansen, and T. Peijs, "High strength and high modulus electrospun nanofibers," Fibers, vol. 2, no. 2, pp. 158-186, 2014.

[123] H. Valentová and J. Stejskal, "Mechanical properties of polyaniline," Synthetic Metals, vol. 160, no. 7-8, pp. 832-834, 2010.

[124] S. J. Pomfret, P. N. Adams, N. P. Comfort, and A. P. Monkman, "Electrical and mechanical properties of polyaniline fibres produced by a one-step wet spinning process," Polymer, vol. 41, no. 6, pp. 2265-2269, 2000.

[125] W. Zhu, F. Masood, J. O’Brien, and L. G. Zhang, "Highly aligned nanocomposite scaffolds by electrospinning and electrospraying for neural tissue regeneration," Nanomedicine: Nanotechnology, Biology, and Medicine, vol. 11, no. 3, pp. 693-704, 2015.

[126] K. T. Shalumon and J. P. Chen, "Scaffold-based drug delivery for cartilage tissue regeneration," Current Pharmaceutical Design, vol. 21, no. 15, pp. 1979-1990, 2015.

[127] H. Chen, Y. Liu, Z. Jiang, W. Chen, Y. Yu, and Q. Hu, "Cellscaffold interaction within engineered tissue," Experimental Cell Research, vol. 323, no. 2, pp. 346-351, 2014.

[128] B. Guo and P. X. Ma, "Synthetic biodegradable functional polymers for tissue engineering: a brief review," Science China Chemistry, vol. 57, no. 4, pp. 490-500, 2014.

[129] D. Chen, Y.-E. Miao, and T. Liu, "Electrically conductive polyaniline/polyimide nanofiber membranes prepared via a combination of electrospinning and subsequent in situ polymerization growth," ACS Applied Materials \& Interfaces, vol. 5, no. 4, pp. 1206-1212, 2013.

[130] M.-C. Chen, Y.-C. Sun, and Y.-H. Chen, "Electrically conductive nanofibers with highly oriented structures and their potential application in skeletal muscle tissue engineering," Acta Biomaterialia, vol. 9, no. 3, pp. 5562-5572, 2013.

[131] T. H. Qazi, R. Rai, and A. R. Boccaccini, "Tissue engineering of electrically responsive tissues using polyaniline based polymers: a review," Biomaterials, vol. 35, no. 33, pp. 9068-9086, 2014.

[132] M. Mattioli-Belmonte, G. Giavaresi, G. Biagini et al., "Tailoring biomaterial compatibility: in vivo tissue response versus in vitro cell behavior," International Journal of Artificial Organs, vol. 26, no. 12, pp. 1077-1085, 2003.

[133] T. J. Rivers, T. W. Hudson, and C. E. Schmidt, "Synthesis of a novel, biodegradable electrically conducting polymer for biomedical applications," Advanced Functional Materials, vol. 12, no. 1, pp. 33-37, 2002.

[134] N. K. Guimard, N. Gomez, and C. E. Schmidt, "Conducting polymers in biomedical engineering," Progress in Polymer Science, vol. 32, no. 8-9, pp. 876-921, 2007.

[135] A. Guiseppi-Elie, "Electroconductive hydrogels: synthesis, characterization and biomedical applications," Biomaterials, vol. 31, no. 10, pp. 2701-2716, 2010.

[136] A. N. Zelikin, D. M. Lynn, J. Farhadi, I. Martin, V. Shastri, and R. Langer, "Erodible conducting polymers for potential biomedical applications," Angewandte Chemie-International Edition, vol. 41, no. 1, pp. 141-144, 2002.

[137] B. K. Gu, M. S. Kim, C. M. Kang, J.-I. Kim, S. J. Park, and C.-H. Kim, "Fabrication of conductive polymer-based nanofiber scaffolds for tissue engineering applications," Journal of Nanoscience and Nanotechnology, vol. 14, no. 10, pp. 76217626, 2014.

[138] T. H. Qazi, R. Rai, D. Dippold et al., "Development and characterization of novel electrically conductive PANI-PGS composites for cardiac tissue engineering applications," Acta Biomaterialia, vol. 10, no. 6, pp. 2434-2445, 2014.

[139] F. F. Azhar, A. Olad, and R. Salehi, "Fabrication and characterization of chitosan-gelatin/nanohydroxyapatite-polyaniline composite with potential application in tissue engineering scaffolds," Designed Monomers and Polymers, vol. 17, no. 7, pp. 654-667, 2014.

[140] P. H. S. Picciani, E. S. Medeiros, Z. Pan, W. J. Orts, L. H. C. Mattoso, and B. G. Soares, "Development of conducting polyaniline/poly(lactic acid) nanofibers by electrospinning," Journal of Applied Polymer Science, vol. 112, no. 2, pp. 744-753, 2009.

[141] M. P. Prabhakaran, L. Ghasemi-Mobarakeh, and S. Ramakrishna, "Electrospun composite nanofibers for tissue regeneration," Journal of Nanoscience and Nanotechnology, vol. 11, no. 4, pp. 3039-3057, 2011.

[142] L. Shadi, M. Karimi, and A. A. Entezami, "Preparation of electroactive nanofibers of star-shaped polycaprolactone/ polyaniline blends," Colloid and Polymer Science, vol. 293, no. 2, pp. 481-491, 2015.

[143] M. Li, Y. Guo, Y. Wei, A. G. MacDiarmid, and P. I. Lelkes, "Electrospinning polyaniline-contained gelatin nanofibers for tissue engineering applications," Biomaterials, vol. 27, no. 13, pp. 2705-2715, 2006.

[144] W.-H. Zimmermann and T. Eschenhagen, "Cardiac tissue engineering for replacement therapy," Heart Failure Reviews, vol. 8, no. 3, pp. 259-269, 2003. 
[145] T. Shimizu, M. Yamato, A. Kikuchi, and T. Okano, "Cell sheet engineering for myocardial tissue reconstruction," Biomaterials, vol. 24, no. 13, pp. 2309-2316, 2003.

[146] L. Ghasemi-Mobarakeh, M. P. Prabhakaran, M. Morshed et al., "Application of conductive polymers, scaffolds and electrical stimulation for nerve tissue engineering," Journal of Tissue Engineering and Regenerative Medicine, vol. 5, no. 4, pp. e17-e35, 2011.

[147] S. H. Ku, S. H. Lee, and C. B. Park, "Synergic effects of nanofiber alignment and electroactivity on myoblast differentiation," Biomaterials, vol. 33, no. 26, pp. 6098-6104, 2012.

[148] P. Gentile, D. Bellucci, A. Sola, C. Mattu, V. Cannillo, and G. Ciardelli, "Composite scaffolds for controlled drug release: role of the polyurethane nanoparticles on the physical properties and cell behaviour," Journal of the Mechanical Behavior of Biomedical Materials, vol. 44, pp. 53-60, 2015.

[149] X. Wang, Y. Yuan, X. Huang, and T. Yue, "Controlled release of protein from core-shell nanofibers prepared by emulsion electrospinning based on green chemical," Journal of Applied Polymer Science, vol. 132, no. 16, Article ID 41811, 2015.

[150] H. Hezaveh and I. I. Muhamad, "Controlled drug release via minimization of burst release in $\mathrm{pH}$-response kappacarrageenan/polyvinyl alcohol hydrogels," Chemical Engineering Research and Design, vol. 91, no. 3, pp. 508-519, 2013.

[151] A. Seyfoddin, A. Chan, W. Chen, I. D. Rupenthal, G. I. N. Waterhouse, and D. Svirskis, "Electro-responsive macroporous polypyrrole scaffolds for triggered dexamethasone delivery," European Journal of Pharmaceutics and Biopharmaceutics, vol. 94, pp. 419-426, 2015.

[152] L. Weng and J. Xie, "Smart electrospun nanofibers for controlled drug release: recent advances and new perspectives," Current Pharmaceutical Design, vol. 21, no. 15, pp. 1944-1959, 2015.

[153] M. R. Abidian, D.-H. Kim, and D. C. Martin, "Conductingpolymer nanotubes for controlled drug release," Advanced Materials, vol. 18, no. 4, pp. 405-409, 2006.

[154] D. C. Martin and M. R. Abidian, "Conducting polymer nanotube actuators for precisely controlled release of medicine and bioactive molecules," U.S. Patent no. 8,936,794, U.S. Patent and Trademark Office, Washington, DC, USA, 2015.

[155] J. Huang, S. Virji, B. H. Weiller, and R. B. Kaner, "Nanostructured polyaniline sensors," Chemistry, vol. 10, no. 6, pp. 13141319, 2004.

[156] A. Z. Sadek, C. O. Baker, D. Powell, W. Wlodarski, R. B. Kaner, and K. Kalantar-Zadeh, "Polyaniline nanofiber based surface acoustic wave gas sensors-effect of nanofiber diameter on $\mathrm{H}_{2}$ response," IEEE Sensors Journal, vol. 7, no. 2, pp. 213-218, 2007.

[157] A. Z. Sadek, W. Wlodarski, K. Kalantar-Zadeh, C. Baker, and R. B. Kaner, "Doped and dedoped polyaniline nanofiber based conductometric hydrogen gas sensors," Sensors and Actuators, A: Physical, vol. 139, no. 1-2, pp. 53-57, 2007.

[158] Y. Liao, C. Zhang, Y. Zhang et al., "Carbon nanotube/polyaniline composite nanofibers: facile synthesis and chemosensors," Nano Letters, vol. 11, no. 3, pp. 954-959, 2011.

[159] A. Z. Sadek, W. Wlodarski, K. Shin, R. B. Kaner, and K. Kalantar-zadeh, "A polyaniline $/ \mathrm{WO}_{3}$ nanofiber compositebased $\mathrm{ZnO} / 64^{\circ} \mathrm{YX} \mathrm{LiNbO}_{3}$ SAW hydrogen gas sensor," Synthetic Metals, vol. 158, no. 1-2, pp. 29-32, 2008.

[160] H. Tai, Y. Jiang, G. Xie, J. Yu, and X. Chen, "Fabrication and gas sensitivity of polyaniline-titanium dioxide nanocomposite thin film," Sensors and Actuators, B: Chemical, vol. 125, no. 2, pp. 644-650, 2007.
[161] D. Ofer, R. M. Crooks, and M. S. Wrighton, "Potential dependence of the conductivity of highly oxidized polythiophenes, polypyrroles, and polyaniline: finite windows of high conductivity," Journal of the American Chemical Society, vol. 112, no. 22, pp. 7869-7879, 1990.

[162] A. Mulchandani and N. V. Myung, "Conducting polymer nanowires-based label-free biosensors," Current Opinion in Biotechnology, vol. 22, no. 4, pp. 502-508, 2011.

[163] M. Gerard, A. Chaubey, and B. D. Malhotra, "Application of conducting polymers to biosensors," Biosensors and Bioelectronics, vol. 17, no. 5, pp. 345-359, 2002.

[164] C. Dhand, M. Das, M. Datta, and B. D. Malhotra, "Recent advances in polyaniline based biosensors," Biosensors \& Bioelectronics, vol. 26, no. 6, pp. 2811-2821, 2011.

[165] N. Promphet, P. Rattanarat, R. Rangkupan, O. Chailapakul, and N. Rodthongkum, "An electrochemical sensor based on graphene/polyaniline/polystyrene nanoporous fibers modified electrode for simultaneous determination of lead and cadmium," Sensors and Actuators B: Chemical, vol. 207, part A, pp. 526-534, 2015.

[166] H. J. Sharma, N. D. Sonwane, and S. B. Kondawar, "Electrospun $\mathrm{SnO}_{2} /$ polyaniline composite nanofibers based low temperature hydrogen gas sensor," Fibers and Polymers, vol. 16, no. 7, pp. 1527-1532, 2015.

[167] K. Low, C. B. Horner, C. Li et al., "Composition-dependent sensing mechanism of electrospun conductive polymer composite nanofibers," Sensors and Actuators B: Chemical, vol. 207, pp. 235-242, 2014.

[168] Y. Wen, Y. Li, Y. Si et al., "Ready-to-use strip for l-ascorbic acid visual detection based on polyaniline/polyamide 66 nanofibers/nets membranes," Talanta, vol. 144, pp. 1146-1154, 2015.

[169] C. Li, N. Chartuprayoon, W. Bosze et al., "Electrospun polyaniline/poly(ethylene oxide) composite nanofibers based gas sensor," Electroanalysis, vol. 26, no. 4, pp. 711-722, 2014.

[170] S. B. Kondawar, P. T. Patil, and S. P. Agrawal, "Chemical vapour sensing properties of electrospun nanofibers of polyaniline/ZnO nanocomposites," Advanced Materials Letters, vol. 5, no. 7, pp. 389-395, 2014.

[171] W. Serrano, A. Meléndez, I. Ramos, and N. J. Pinto, "Sensor response of electrospun poly(lactic acid)/polyaniline nanofibers to aliphatic alcohol vapors of varying sizes," in Proceedings of the IEEE 9th Ibero-American Congress on Sensors (IBERSENSOR '14), pp. 1-4, IEEE, Bogotá, Colombia, October 2014.

[172] R. Huang, Y. Z. Long, C. C. Tang, and H. D. Zhang, "Fabrication of nano-branched coaxial polyaniline/polyvinylidene fluoride fibers via electrospinning for strain sensor," Advanced Materials Research, vol. 853, pp. 79-82, 2013.

[173] H. D. Zhang, C. C. Tang, Y. Z. Long et al., "High-sensitivity gas sensors based on arranged polyaniline/PMMA composite fibers," Sensors and Actuators A: Physical, vol. 219, pp. 123-127, 2014.

[174] Y. A. Ismail, J. G. Martínez, and T. F. Otero, "Fibroin/polyaniline microfibrous mat. preparation and electrochemical characterization as reactive sensor," Electrochimica Acta, vol. 123, pp. 501510, 2014.

[175] Z. Pang, J. Fu, L. Luo, F. Huang, and Q. Wei, "Fabrication of $\mathrm{PA} 6 / \mathrm{TiO}_{2} / \mathrm{PANI}$ composite nanofibers by electrospinningelectrospraying for ammonia sensor," Colloids and Surfaces A: Physicochemical and Engineering Aspects, vol. 461, no. 1, pp. 113118, 2014. 
[176] D. Aussawasathien, J.-H. Dong, and L. Dai, "Electrospun polymer nanofiber sensors," Synthetic Metals, vol. 154, no. 1-3, pp. 37-40, 2005.

[177] Q. Lin, Y. Li, and M. Yang, "Polyaniline nanofiber humidity sensor prepared by electrospinning," Sensors and Actuators B: Chemical, vol. 161, no. 1, pp. 967-972, 2012.

[178] Q. Lin, Y. Li, and M. Yang, "Highly sensitive and ultrafast response surface acoustic wave humidity sensor based on electrospun polyaniline/poly(vinyl butyral) nanofibers," Analytica Chimica Acta, vol. 748, pp. 73-80, 2012.

[179] D. Chen, X. Guo, Z. Wang, P. Wang, Y. Chen, and L. Lin, "Polyaniline nanofiber gas sensors by direct-write electrospinning," in Proceedings of the 24th IEEE International Conference on Micro Electro Mechanical Systems (MEMS '11), pp. 1369-1372, IEEE, Cancun, Mexico, January 2011.

[180] A. K. Singh and K. Mandal, "Engineering of high performance supercapacitor electrode based on $\mathrm{Fe}-\mathrm{Ni} / \mathrm{Fe}_{2} \mathrm{O}_{3}-\mathrm{NiO}$ core/shell hybrid nanostructures," Journal of Applied Physics, vol. 117, Article ID 105101, 2015.

[181] Z. Tang, C.-H. Tang, and H. Gong, "A high energy density asymmetric supercapacitor from nano-architectured $\mathrm{Ni}(\mathrm{OH})_{2} /$ carbon nanotube electrodes," Advanced Functional Materials, vol. 22, no. 6, pp. 1272-1278, 2012.

[182] Z. B. Lei, L. Lu, and X. S. Zhao, "The electrocapacitive properties of graphene oxide reduced by urea," Energy and Environmental Science, vol. 5, no. 4, pp. 6391-6399, 2012.

[183] S. Chen, J. Zhu, X. Wu, Q. Han, and X. Wang, "Graphene oxide$\mathrm{Mn}_{\mathrm{o} 2}$ nanocomposites for supercapacitors," ACS Nano, vol. 4, no. 5, pp. 2822-2830, 2010.

[184] C. Yuan, L. Yang, L. Hou et al., "Flexible hybrid paper made of monolayer $\mathrm{Co}_{3} \mathrm{O}_{4}$ microsphere arrays on $\mathrm{rGO} / \mathrm{CNT}$ and their application in electrochemical capacitors," Advanced Functional Materials, vol. 22, no. 12, pp. 2560-2566, 2012.

[185] E. T. Kang, K. G. Neoh, and K. L. Tan, "Polyaniline: a polymer with many interesting intrinsic redox states," Progress in Polymer Science, vol. 23, no. 2, pp. 277-324, 1998.

[186] S. Chaudhari, Y. Sharma, P. S. Archana et al., "Electrospun polyaniline nanofibers web electrodes for supercapacitors," Journal of Applied Polymer Science, vol. 129, no. 4, pp. 1660-1668, 2013.

[187] F. Miao, C. Shao, X. Li et al., "Flexible solid-state supercapacitors based on freestanding electrodes of electrospun polyacrylonitrile@polyaniline core-shell nanofibers," Electrochimica Acta, vol. 176, pp. 293-300, 2015.

[188] Y. Miao, J. Yan, Y. Huang, W. Fan, and T. Liu, "Electrospun polymer nanofiber membrane electrodes and an electrolyte for highly flexible and foldable all-solid-state supercapacitors," RSC Advances, vol. 5, no. 33, pp. 26189-26196, 2015.

[189] A. Rose, N. Raghavan, S. Thangavel, B. U. Maheswari, D. P. Nair, and G. Venugopal, "Investigation of cyclic voltammetry of graphene oxide/polyaniline/polyvinylidene fluoride nanofibers prepared via electrospinning," Materials Science in Semiconductor Processing, vol. 31, pp. 281-286, 2015.

[190] W. F. Mak, G. Wee, V. Aravindan, N. Gupta, S. G. Mhaisalkar, and S. Madhavi, "High-energy density asymmetric supercapacitor based on electrospun vanadium pentoxide and polyaniline nanofibers in aqueous electrolyte," Journal of the Electrochemical Society, vol. 159, no. 9, pp. A1481-A1488, 2012.

[191] J. Burschka, N. Pellet, S.-J. Moon et al., "Sequential deposition as a route to high-performance perovskite-sensitized solar cells," Nature, vol. 499, no. 7458, pp. 316-319, 2013.
[192] S. Pan, Z. Yang, P. Chen, J. Deng, H. Li, and H. S. Peng, "Wearable solar cells by stacking textile electrodes," Angewandte Chemie-International Edition, vol. 53, no. 24, pp. 6110-6114, 2014.

[193] M. K. Nazeeruddin, F. De Angelis, S. Fantacci et al., "Combined experimental and DFT-TDDFT computational study of photoelectrochemical cell ruthenium sensitizers," Journal of the American Chemical Society, vol. 127, no. 48, pp. 16835-16847, 2005.

[194] N. Papageorgiou, W. F. Maier, and M. Grätzel, "An iodine/ triiodide reduction electrocatalyst for aqueous and organic media," Journal of the Electrochemical Society, vol. 144, no. 3, pp. 876-884, 1997.

[195] Y.-L. Lee, C.-L. Chen, L.-W. Chong, C.-H. Chen, Y.-F. Liu, and C.-F. Chi, "A platinum counter electrode with high electrochemical activity and high transparency for dye-sensitized solar cells," Electrochemistry Communications, vol. 12, no. 11, pp.1662$1665,2010$.

[196] X. Yin, Z. S. Xue, and B. Liu, "Electrophoretic deposition of Pt nanoparticles on plastic substrates as counter electrode for flexible dye-sensitized solar cells," Journal of Power Sources, vol. 196, no. 4, pp. 2422-2426, 2011.

[197] Y. Duan, Y. Chen, Q. Tang et al., "A dye-sensitized solar cell having polyaniline species in each component with $3.1 \%$ efficiency," Journal of Power Sources, vol. 284, pp. 178-185, 2015.

[198] S. Peng, P. Zhu, Y. Wu, S. G. Mhaisalkar, and S. Ramakrishna, "Electrospun conductive polyaniline-polylactic acid composite nanofibers as counter electrodes for rigid and flexible dyesensitized solar cells," RSC Advances, vol. 2, no. 2, pp. 652-657, 2012.

[199] S. Ebrahim, M. Soliman, M. Anas, M. Hafez, and T. M. Abdel-Fattah, "Dye-sensitized solar cell based on polyaniline/multiwalled carbon nanotubes counter electrode," International Journal of Photoenergy, vol. 2013, Article ID 906820, 6 pages, 2013. 

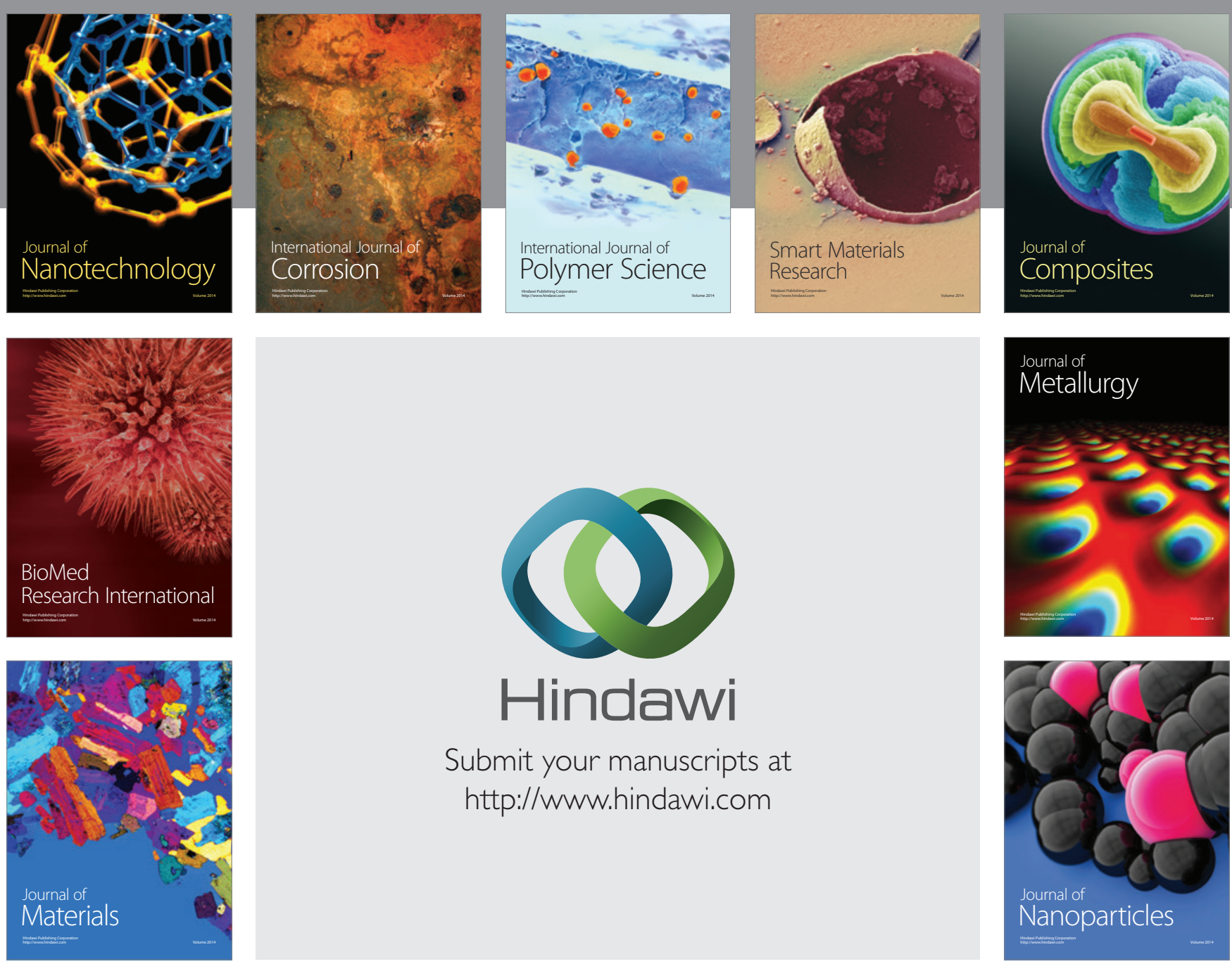

Submit your manuscripts at http://www.hindawi.com
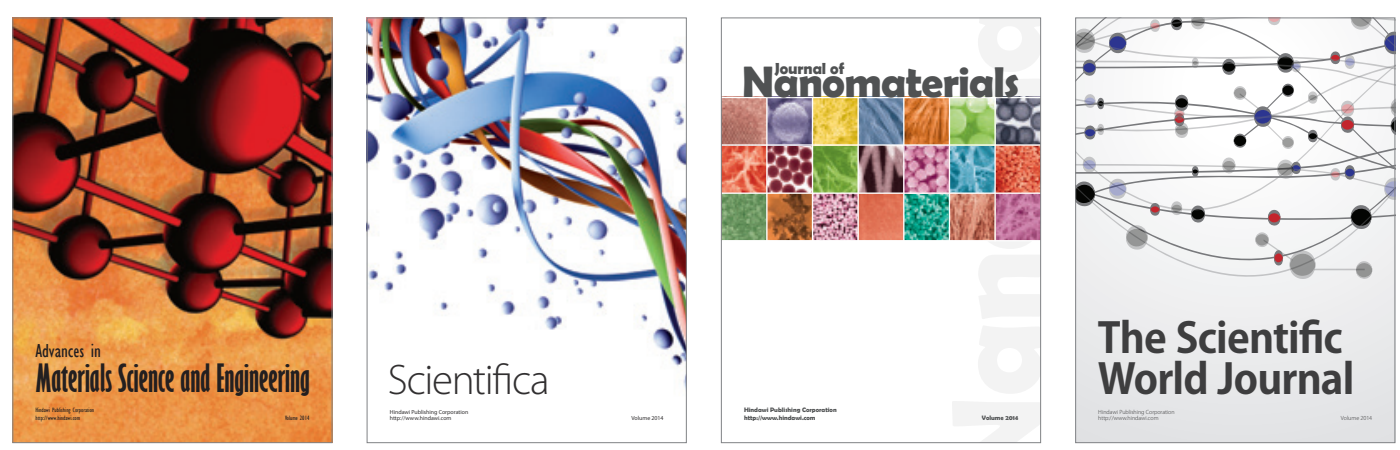

\section{The Scientific World Journal}
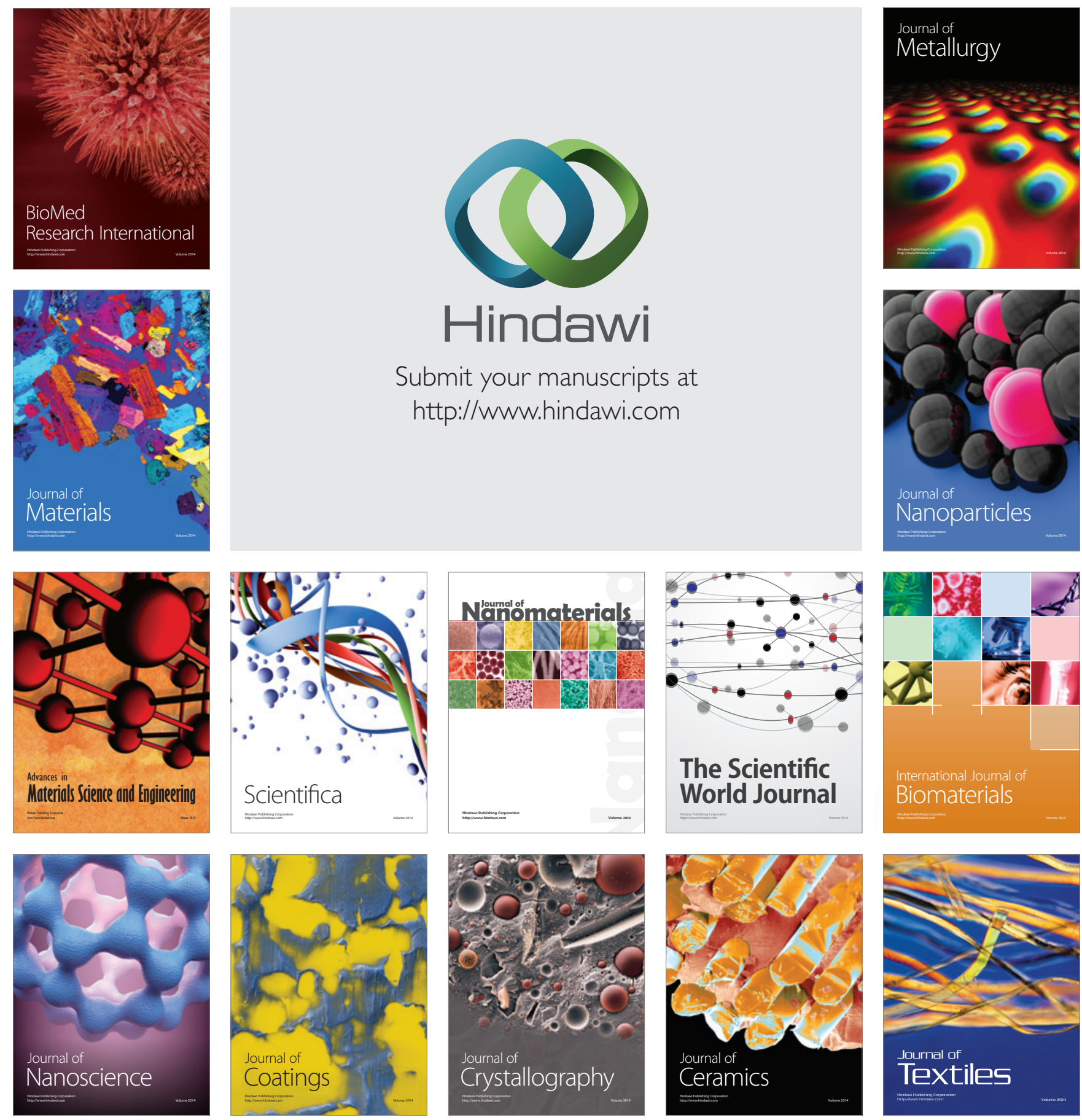